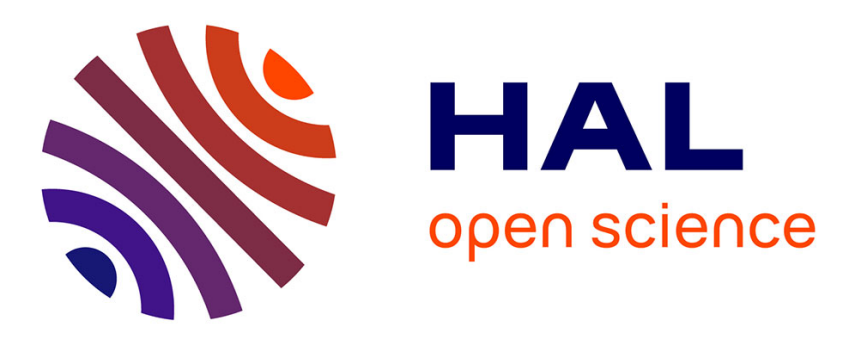

\title{
Design of a reliable logistics network with hub disruption under uncertainty
}

Mehrdad Mohammadi, Reza Tavakkoli-Moghaddam, Ali Siadat, Jean-Yves Dantan

\section{- To cite this version:}

Mehrdad Mohammadi, Reza Tavakkoli-Moghaddam, Ali Siadat, Jean-Yves Dantan. Design of a reliable logistics network with hub disruption under uncertainty. Applied Mathematical Modelling, 2016, 40 (9-10), pp.1-22. 10.1016/j.apm.2016.01.011 . hal-01794858

\section{HAL Id: hal-01794858 https://imt-atlantique.hal.science/hal-01794858}

Submitted on 25 Mar 2020

HAL is a multi-disciplinary open access archive for the deposit and dissemination of scientific research documents, whether they are published or not. The documents may come from teaching and research institutions in France or abroad, or from public or private research centers.
L'archive ouverte pluridisciplinaire $\mathbf{H A L}$, est destinée au dépôt et à la diffusion de documents scientifiques de niveau recherche, publiés ou non, émanant des établissements d'enseignement et de recherche français ou étrangers, des laboratoires publics ou privés. 


\title{
Design of a reliable logistics network with hub disruption under uncertainty
}

\author{
Mehrdad Mohammadi ${ }^{\mathrm{a}, \mathrm{b}, \mathrm{c}, *}$, Reza Tavakkoli-Moghaddamª, Ali Siadat ${ }^{\mathrm{b}}$, \\ Jean-Yves Dantan ${ }^{\mathrm{b}}$ \\ a School of Industrial Engineering, College of Engineering, University of Tehran, Tehran, Iran \\ ${ }^{\mathrm{b}}$ LCFC Laboratory, Arts et Métiers ParisTech, 4 rue Augustin Fresnel, 57078 Metz, France \\ ${ }^{\mathrm{c}}$ Ecole des Mines de Saint-Etienne, Department of Manufacturing Sciences and Logistics, CMP, CNRS UMR 6158 LIMOS, 880 avenue de \\ Mimet, 13541 Gardanne, France
}

\begin{abstract}
A B S T R A C T
In this study, we design a reliable logistics network based on a hub location problem, which is less sensitive to disruption and it performs efficiently when disruption occurs. A new mixed-integer programming model is proposed to minimize the total sum of the nominal and expected failure costs. This model considers complete and partial disruption at hubs. In addition, we propose a new hybrid meta-heuristic algorithm based on genetic and imperialist competitive algorithms. We compare the performance of the proposed algorithm with a new lower bound method in terms of the CPU time and solution quality. Furthermore, we conclude that a considerable improvement in the reliability of the network can be achieved with only a slight increase in the total cost. Finally, we demonstrate that the networks designed using our model are less conservative and more robust to disruption compared with those designed based on other robustness measures.
\end{abstract}

\section{Introduction}

Strategic decisions about the locations of new facilities, boosting distribution networks, and cooperating with new parties are required in most industries. Industrial companies must locate manufacturing facilities as well as distribution centers, which need to be positioned near retail outlets. Thus, the success of these industries depends greatly on the quality of the location-related decisions. Similarly, governments must make decisions about the locations of hospitals, police offices, schools, fire stations, and other facilities. In each case, the location of a facility has a significant effect on the quality of the logistics network.

In general, logistics networks are responsible for transferring and distributing flows between a set of origin and destination nodes through a number of intermediate facilities. In most previous studies of this problem, it has been assumed that the facilities are always available. However, logistics networks designed according to this assumption may encounter serious problems if they fail to consider disruption in any component of the network, and thus they would become ineffective when the facilities are subject to failure. Recently, logistics networks have become increasingly complicated due to their size, the services required, the nature of customer assignments, the utilization of several components, and associated network flows. 
Thus, a small failure in a component may propagate throughout the system and cause a huge inconsistency in the network, which further imposes a tremendous cost, with irrecoverable or difficult to recover effects on the network [1].

Facilities might fail due to several intentional/unintentional reasons such as natural disasters, equipment breakdowns, supplier cutouts, explosions due to process hazards, labor strikes, and terrorist attacks. Peng et al. [2] highlighted the shortterm and long-term effects of disruption in logistics networks such as increases in costs and the time of transportation, delivery delays, and inventory shortages, as well as negative financial effects. Thus, enterprises must aim to design a reliable logistics network in order to cope with these short- and long-term inefficiencies.

In this study, we consider the problem of designing a logistics network as a hub location problem (HLP), which comprises origin, hub, and destination nodes. In a hub network, a set of intermediate nodes are located as hubs, and origin and destination nodes (spokes) are allocated to these hubs. Hubs are responsible for consolidating, transferring, and distributing flows through the network to obtain economic profits. After a hub network has been designed, it can be very difficult and costly to modify the design. Therefore, it is necessary to design a reliable logistics network that remains available and efficient in the presence of many types of disruptions.

In classical HLPs, it is usually considered that the topology of the hub network is not changed after the hubs have been located and spokes are allocated to the hubs. It is also assumed that the hub nodes are always available and no event can disrupt their performance. However, these are not valid assumptions if the located hubs are subject to failure and they may become unavailable due to disruptions caused by intentional or unintentional reasons. Disruption at hubs can belong to two types: complete and partial disruptions.

When a hub node is completely disrupted, the hub becomes unavailable and the spokes originally allocated to it must be reallocated to other (operational) hub nodes, which usually incurs higher connection costs (i.e., re-allocation cost). During partial disruption, although the hub node may be still available, the service rate or the capacity of the hub is degraded to a lower level. If service rate degradation occurs, the hubs become congested and the incoming flow must wait to be processed. During capacity degradation, the capacity of the hub is degraded to a lower level and the hub is unable to process the entire arrival flow (i.e., the capacity of the hub is decreased). Therefore, disruptions in a hub network can greatly affect the performance of the logistics network.

The main aim of this study is to introduce a new mathematical model for designing a reliable logistics network based on a HLP under disruption, where the flows should be transferred between each pair of origin-destination nodes via a given set of links. A brief survey of previous research (Section 2) shows that few previous studies have addressed the design of a reliable hub network, where hubs are subject to complete and partial disruption, and thus they may fail to process the flows. In addition, in real settings, after designing a hub network, some parameters (e.g., demand, distance, time, and cost) in the problem may change due to uncertainty, but most previous studies have not adequately addressed how uncertain parameters might affect the network design. The uncertainty in parameters may come from a variety of sources, but collecting historical data is as difficult as estimating the probabilities of the parameters. Hence, we utilize the robust optimization approach proposed by Peng et al. [2], which provides an alternative method for coping with uncertainty but without requiring probabilistic information about the input parameters.

The main contributions of this study are as follows.

- We design and model a new reliable logistics network as a HLP by considering the possibility of disruption in the hub facilities.

- We consider the effects of two different types of disruptions: (1) hub availability, i.e., complete disruption; and (2) hub capacity, i.e., partial disruption.

- We develop a service level constraint that considers disruption of the capacity.

- A new hybrid solution approach is proposed by combining a number of efficient solution approaches from recent research, i.e., stochastic programming and robust optimization.

- An efficient lower bound approach is proposed to find a near-optimal solution in a reasonable amount of computational time with few gaps.

- We propose a new hybrid self-adaptive meta-heuristic algorithm based on genetic algorithms and imperialist competitive algorithms (ICAs).

The remainder of this paper is organized as follows. Section 2 describes previous research in the area of hub networks by considering disruptions in the hub facilities. Section 3 explains the problem and the mathematical formulation, and the robust optimization approach is presented in Section 4. The new lower bound and meta-heuristic methods are introduced in Section 5. Computational results are provided in Section 6. Finally, we give our conclusions in Section 7.

\section{Literature review}

Most previous studies of disruption in networks are based on the classical p-median [3] and uncapacitated fixed-charge location problems [4]. Both of these problems locate facilities and assign customers to the located facilities to minimize the total transportation (and/or construction) cost, where all facilities are assumed to be totally available and reliable. Some recent studies have considered facility location in the presence of random disruptions and interested readers may refer to a comprehensive review by Snyder [5]. Most previous studies in this area considered disruptions in a facility location problem, so most of this section deals with studies related to the facility location problem in the presence of disruption. 
One of the first mathematical models used to study a reliable facility location problem was presented by Drezner and Wesolowsky [6], where an unreliable $p$-median and $(p, q)$-center location problems were considered and facilities had a given probability of becoming inactive. Snyder and Daskin [7] presented two reliability models (i.e., reliable $p$-median and reliable uncapacitated fixed-charge location) for facility location, where each customer is assigned to a primary facility and a number of backup facilities, and at least one facility must be available. If the current facility fails, the customer is served by the next available backup facility. They also considered that the probabilities of failure are equal and mutually independent for all facilities. Cui et al. [1], Berman et al. [8], Li and Ouyang [9], Lim et al. [10,11], Shen et al. [12], Snyder and Daskin [13], and Zhan et al. [14] all described models similar to Snyder and Daskin [7], but they relaxed the uniform disruption probability assumption using different modeling approaches.

Li and Ouyang [9] studied facility location using a three-echelon supply chain problem by considering random disruptions for both suppliers and retailers. Their model determines the optimal locations of retailers, customer assignments, and inventory policy. Peng et al. [2] introduced the p-robustness criterion, which allows a designed network to perform well in both disrupted and normal conditions. They proposed a hybrid meta-heuristic algorithm based on a genetic algorithm, local improvement, and the shortest augmenting path method. According to different numerical experiments, they demonstrated the superior performance of the proposed heuristic compared with CPLEX in terms of the computational time, while still obtaining an excellent quality solution. They also showed that the reliability of the network could be improved substantially with only a slight increase in cost. Finally, they found that their model produced less conservative solutions than those generated by common robustness measures.

It should be noted that Peng et al. [2] defined some specific disruption scenarios and considered that hubs are absolutely disrupted in each scenario. In the present study, we also consider a set of disruption scenarios and the probability that each hub is disrupted in each scenario. We believe that our assumption is more applicable when decision makers cannot determine whether there is any disruption in each scenario. In addition, we employ their proposed robust method to cope with the uncertainties of the input parameters (e.g., costs, disruption probabilities, and capacity degradation factors).

Li et al. [15] presented two related models (i.e., reliable $p$-median and reliable uncapacitated fixed-charge location) for designing reliable distribution networks. Both models were formulated by nonlinear integer programming and heterogeneous facility failure probabilities were also considered, with one layer of supplier backup and facility fortification within a finite budget. The NP-hardness of these models was also proved.

To the best of our knowledge, only three previous studies have addressed the hub network design problem by considering hub disruptions. Parvaresh et al. [16] formulated a bi-level multiple allocation $p$-hub median problem under intentional disruptions by a bi-level model with bi-objective functions at an upper level and a single objective at a lower level. In their model, the leader aims to identify the locations of hubs such that the normal and worst-case transportation costs are minimized while considering the normal and failure conditions. Finally, the worst-case scenario is modeled at a lower level, where the follower's objective is to identify the hubs that would reduce the service efficiency most if they are lost. They also developed two multi-objective meta-heuristics based on simulated annealing and tabu search to solve their proposed model. In a similar study, Parvaresh et al. [17] developed a multiple allocation $p$-hub median problem under intentional disruptions using different definitions of the failure probability for a hub compared with their previous study.

More recently, Mohammadi et al. [18] proposed a bi-objective stochastic $p$-hub center problem to investigate the effect of delivery service requirements on the configuration of a hub-and-spoke network with fuzzy parameters, where the hub operational nodes may be disrupted and fail to operate shipments. They considered that the delivery time of the shipments is the sum of the transit time over the links and the time spent at the hubs. They calculated the time spent waiting for the fuzzy arrival of shipments by utilizing a fuzzy $\mathrm{M} / \mathrm{M} / 1$ queuing system. They also assumed that the queuing system in the hubs is degraded stochastically and then restored to its performance level.

\section{Problem description and formulation}

In this study, we design a logistics network with facility disruptions based on a hub location network. This problem generalizes the classical HLP by making decisions regarding the locations of hubs, the allocation of origin and destination nodes to the located hub, and routing the flows through the network. Thus, we propose a new mixed-integer nonlinear programming model for designing a reliable hub network. Hubs are affected by two types of disruption, i.e., disruptions in hub facilities and disruption in the designed capacity of hubs. A new assumption that includes the probability of failure for the hubs is considered for hub disruption. When hub disruption occurs, the hub becomes unavailable and no origin and destination nodes are served. In this situation, backup hubs are needed to route the flows. Therefore, a set of potential hubs are located and the origin and destination nodes are allocated to the hubs level by level (i.e., backup hubs). Thus, for a non-hub node, after the corresponding hub in level one is disrupted, the non-hub node is reallocated to the backup hub in level two. This reallocation policy is repeated for the non-hub node after a disruption occurs at a higher level and it is continued after the non-hub node has been allocated to a dummy non-failable hub.

By contrast, during capacity disruption at a hub, the hub is available and only the size of the flow that enters the hubs must be decreased. In addition, the capacity of a hub is subject to stochastic degradation, so we consider a certain probability that a traffic flow entering a hub will exceed the actual capacity and disrupt the flow process. To address this issue, it is desirable to ensure that the probability of such an occurrence is lower than a specified or satisfactory level. 


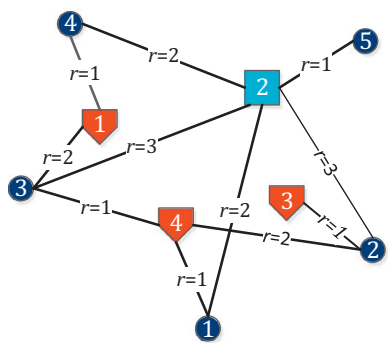

a) Allocation Structure

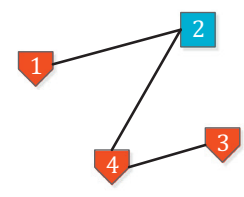

b) Tree Structure

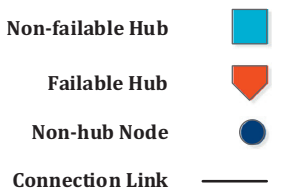

Connection Link

Fig. 1. Logistics of the proposed RHLP.

In terms of the structure of the hub network, most studies of HLPs have considered that a hub network is a complete graph where all of the hubs are fully interconnected. This assumption makes it easier to model the HLPs, but there is some justification for designing incomplete hub networks for various applications. For example, in distribution networks, sending separate trucks from a distribution center (i.e., hub) to all other distribution centers is expensive in terms of the fixed cost of using a high number of trucks. Instead, using the same trucks with sufficient capacity to visit more than one distribution center may decrease the total investment cost considerably. Similarly, in airline passenger transportation, an airline company may route and force its flights to pass through a large number of destinations because the requirement for more separate aircraft and air staff at each destination imposes higher congestion and investment costs in airports and air networks for the company [19]. A suitable structure for incomplete HLPs is a tree structure, which places a single allocation $p$-HLP where $p$ hubs must be connected using a (non-directed) tree [20]. Thus, we consider a tree structure for the hub network. In order to understand the HLP model easily, Fig. 1 illustrates the logistics network with a reallocation policy and the tree structure of the hub network. As shown in Fig. 1a, non-hub node 1 is allocated to failable hub 4 at the first level (i.e., $r=1$ ) and after the hub 4 is disrupted, the non-hub node is allocated to dummy non-failable hub 2 at level 2 (i.e., $r=2$ ), and the reallocation policy is then stopped. The tree structure of the hub network is shown in Fig. 1b, where the hubs are connected by a tree-shape graph.

In the following subsections, we formulate the single-objective reliable HLP (SRHLP) to minimize the sum of the nominal transportation and expected failure costs. First, we develop a service level that considers the hub capacity reliability and we then introduce the assumptions of the problem, before describing the initial nonlinear model of the SRHLP.

\subsection{Hub capacity reliability}

As mentioned in Section 1, the capacity of a hub may be subject to stochastic degradation, where the flows entering the hub will exceed the designed capacity by a certain probability. It is desirable to ensure that the probability of such an occurrence is lower than a specified or satisfactory level. We introduce the hub capacity reliability as the probability of the flow entering a hub exceeding its capacity, which is referred to as the capacity exceedance probability $\eta$. The hub capacity reliability can be formulated mathematically as:

$$
P\{\Gamma \leq E F\} \leq \eta,
$$

where $E F$ and $\Gamma$ are the flows entering the hub and the capacity of the hub, respectively. It should be noted that the hub capacity reliability requirement can differ for different hubs. In inequality (1), $\Gamma$ is a variable specified by a particular probability density function. The left-hand side of inequality (1) can be considered as a cumulative distribution function (CDF) of $\Gamma$, which is written as follows.

$$
F_{\Gamma}(E F)=P\{\Gamma \leq E F\}
$$

Using this equation, inequality (1) can be rewritten as:

$$
F_{\Gamma}(E F) \leq \eta \text {. }
$$

The CDFs are monotonic one-to-one functions, so we can take the inverse of Eq. (3) and write the following equation.

$$
E F \leq F_{\Gamma}^{-1}(\eta) .
$$

By specifying the CDF of the hub capacity $\Gamma$ and the acceptable capacity exceedance probability $\eta$, Eq. (4) becomes a deterministic constraint. For simplicity, we assume that the hub capacity follows a uniform distribution defined by an upper bound (i.e., design capacity) and a lower bound (i.e., worst-degraded capacity). Generalizing the consideration to other probability distributions (e.g., Gamma and truncated Gamma) can be accomplished with the Mellin Transform technique, as 
discussed in [21]. It should be noted that several studies [21-24] suggest that a uniform distribution is more applicable to the transportation domain when affected by disruption.

It should be mentioned that other distribution functions (e.g., Gamma and truncated Gamma) can be used to model the capacity degradation. Furthermore, we consider that the lower bound is a fraction $\theta$ of the design capacity. For a uniform distribution, the inverse $\mathrm{CDF}$ of $\Gamma$ can be written as:

$$
F_{\Gamma}^{-1}(\eta)=\theta \bar{\Gamma}+\eta \bar{\Gamma}(1-\theta)=\bar{\Gamma}[\theta+\eta(1-\theta)],
$$

where $\bar{\Gamma}$ is the design capacity of the hub, which has a deterministic value. By putting Eq. (5) into inequality (4), we obtain the following hub capacity reliability.

$$
E F \leq \bar{\Gamma}[\theta+\eta(1-\theta)]
$$

\subsection{Assumptions}

The SRHLP is formulated under some specific assumptions as follows.

- The number of hubs that should be located is given.

- Each non-hub node can be allocated to exactly one hub at each level (i.e., single allocation).

- The graph of hubs is formed as an indirect tree structure.

- Locating the hubs incurs a fixed cost.

- A fixed failure probability is associated with each hub.

\subsection{Mathematical formulation of the SRHLP} Sets

In this section, we first present the notations for the problem and the mathematical formulation is then proposed.

$$
\begin{array}{ll}
i, j=\{1, \ldots, I\} & \text { set of nodes } \\
k, l, m, n=\{1, \ldots, K+1\} & \text { set of hubs where the }(K+1) \text { th hub is a dummy non-failable hub } \\
r=\{1, \ldots, R\} & \text { set of allocation levels }
\end{array}
$$

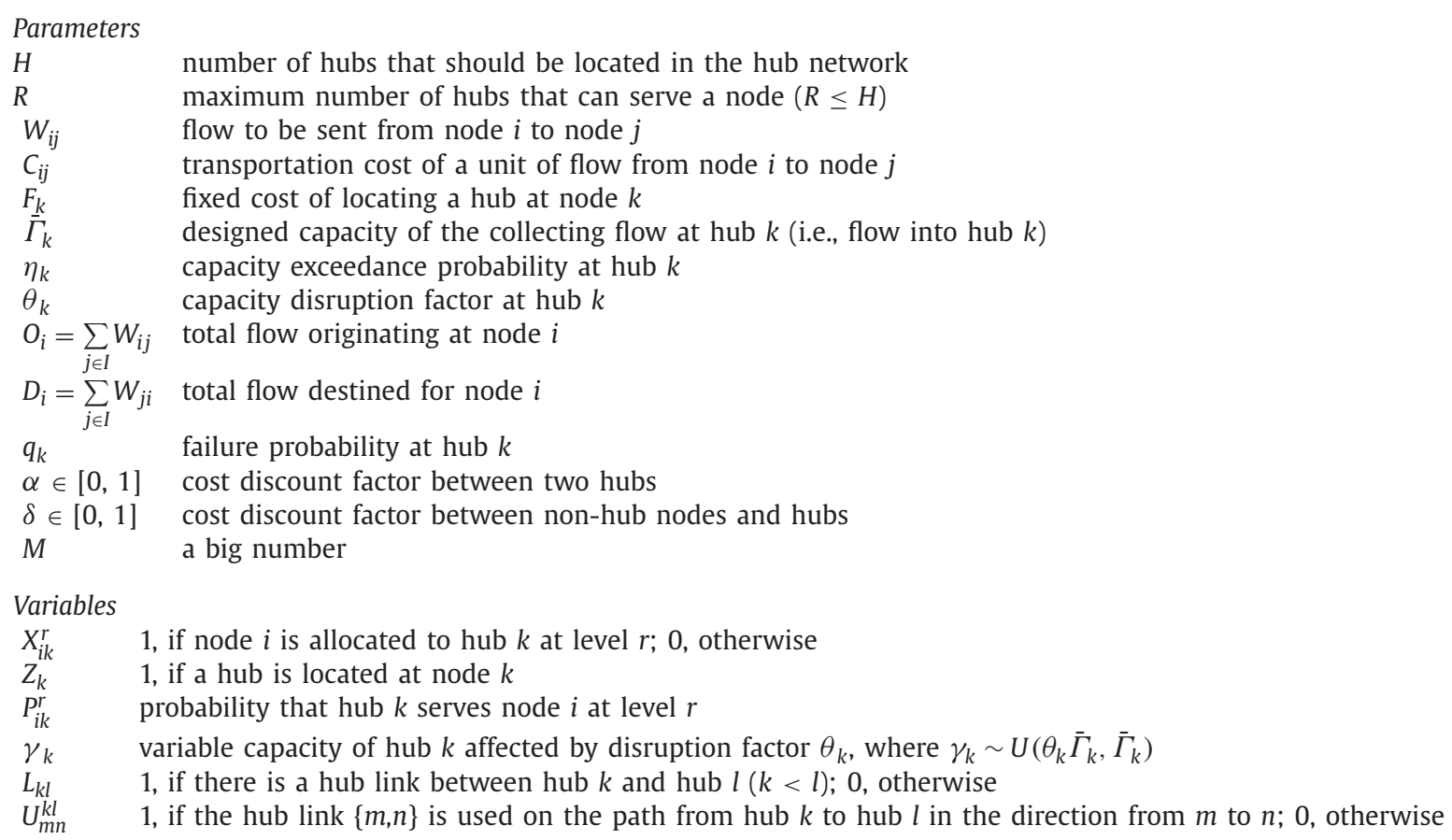

Remark. A "level- $r$ " assignment is one for which $r$ failable hubs that are open. If $r=0$, this is a primary assignment; otherwise, it is a backup assignment. Each non-hub node $j$ has a level- $r$ assignment for each $r=0,1, \ldots, R-1$, unless $j$ is assigned to a level- $l$ hub that is dummy non-failable, where $l<r$. In other words, non-hub node $j$ is allocated to one hub at level 0 , another hub at level 1 , and so on until $j$ is allocated to all of the open hubs at some level, or $j$ is allocated to a dummy non-failable hub [7]. 
By employing the specified notations, the proposed SRHLP is presented as follows.

$$
\begin{aligned}
& \min \sum_{k=1}^{K} F_{k} Z_{k}+\sum_{i=1}^{I} \sum_{k=1}^{K+1} \sum_{r=1}^{R} \delta C_{i k}\left(O_{i}+D_{i}\right) P_{i k}^{r} X_{i k}^{r} \\
& +\sum_{m=1}^{K+1} \sum_{n=1}^{K+1} \alpha C_{m n}\left[\sum_{i=1}^{I} \sum_{\substack{j=1 \\
j \neq i}}^{I} \sum_{k=1}^{I} \sum_{\substack{l=1 \\
l \neq k}}^{K+1} \sum_{r=1}^{R} \sum_{s=1}^{R} W_{i j} U_{m n}^{k l} P_{i m}^{r} X_{i m}^{r} P_{j n}^{s} X_{j n}^{S}\right],
\end{aligned}
$$

s.t.

$$
\begin{aligned}
& \sum_{k=1}^{K} X_{i k}^{r}+\sum_{s=1}^{r} X_{i(K+1)}^{s}=1 \quad \forall i \in I, r \in\{1, \ldots, R\}, \\
& \sum_{r=1}^{R} X_{i(K+1)}^{r}=1 \quad \forall i \in I, \\
& P_{i k}^{1}=1-q_{k} \quad \forall i \in I, k \in\{1, \ldots, K+1\}, \\
& P_{i l}^{r}=\left(1-q_{l}\right) \sum_{k=1}^{K} \frac{q_{k}}{1-q_{k}} P_{i k}^{r-1} X_{i k}^{r-1} \quad \forall i \in I, l \in\{1, \ldots, K+1\}, r \in\{2, \ldots, R\}, \\
& \sum_{k=1}^{K} Z_{k}=H, \\
& Z_{K+1}=1,
\end{aligned}
$$$$
\sum_{r=1}^{R} X_{i k}^{r} \leq Z_{k} \quad \forall i \in I, k \in\{1, \ldots, K\}
$$

$\sum_{m=1}^{K+1} \sum_{i=1}^{I} \sum_{\substack{j=1 \\ j \neq i}}^{I} \sum_{k=1}^{I} \sum_{\substack{l=1 \\ l \neq k}}^{K+1} \sum_{r=1}^{R} \sum_{s=1}^{R} W_{i j} U_{m n}^{k l} P_{i m}^{r} X_{i m}^{r} P_{j n}^{s} X_{j n}^{s} \leq \bar{\Gamma}_{n}\left[\theta_{n}\left(1-\eta_{n}\right)+\eta_{n}\right] \quad \forall n \in\{1, \ldots, K\}$,

$\sum_{k=1}^{K+1} \sum_{\substack{l=1 \\ k<l}}^{K+1} L_{k l}=H-1$

$L_{k l} \leq \frac{1}{2}\left(Z_{k}+Z_{l}\right) \quad \forall k, l \in\{1, \ldots, K+1\}, k<l$,

$U_{m n}^{k l}+U_{n m}^{k l} \leq \frac{1}{3}\left(L_{m n}+Z_{k}+Z_{l}\right) \quad \forall m, n, k, l \in\{1, \ldots, K+1\}, m<n, k \neq l$,

$\sum_{\substack{n=1 \\ n \neq m}}^{K+1} U_{m n}^{m l} \geq Z_{m}+Z_{l}-1 \quad \forall m, l \in\{1, \ldots, K+1\}, m \neq l$

$\sum_{\substack{n=1 \\ n \neq m}}^{K+1} U_{n m}^{k l}-\sum_{\substack{n=1 \\ n \neq m}}^{K+1} U_{m n}^{k l}=0 \quad \forall m, k, l \in\{1, \ldots, K+1\}, m \neq k, m \neq l, k \neq l$,

$\sum_{\substack{n=1 \\ n \neq m}}^{K+1} U_{n m}^{k m} \geq Z_{m}+Z_{k}-1 \quad \forall m, k \in\{1, \ldots, K+1\}, m \neq k$ 


$$
\begin{aligned}
& X_{i k}^{r}, Z_{k}, U_{m n}^{k l}, L_{k l} \in\{0,1\} \quad \forall i \in I ; k, m, n \in\{1, \ldots, K+1\} ; r \in R, \\
& P_{i k}^{r} \geq 0 \quad \forall i \in I ; k, l \in\{1, \ldots, K+1\} ; r \in R .
\end{aligned}
$$

Objective function (7) is the sum of the fixed and expected transportation costs. Eq. (8) ensures that for each non-hub node $i$ and each level $r$, either $i$ is allocated to a regular hub at level $r$ or it is assigned to the reliable hub $K+1$ at certain level $s \leq r$ (taking $\sum_{s=1}^{r} X_{i, K+1}^{s}=0$ if $r=1$ ). Constraint (9) ensures that each non-hub node is allocated to the dummy non-failable hub at a certain level. Constraint (10) shows that the probability that hub $k$ serves non-hub node $i$ at level 1 is simply the probability that $k$ is not disrupted. Constraint (11) is the "transitional probability" equation, which shows that the probability of hub $l$ serving non-hub node $i$ at level $r$ (i.e., $\left.P_{i l}^{r}\right)$ is calculated when hub $l$ remains open (i.e., with probability $\left.\left(1-q_{l}\right)\right)$ and other hubs to which node $i$ has been previously allocated in lower levels than $r$ have been disrupted. Constraint (12) ensures that the number of hubs should be equal to the given value $H$. Eq. (13) requires that the dummy non-failable hub $K+1$ is open. Constraint (14) forces non-hub nodes to be allocated to valid hubs. Constraint (15) limits the amount of flows entering the hubs. According to constraint (16), the variables $L$ define a tree since $H-1$ edges are selected. In order to establish a hub link $\{k, l\}$, both end nodes (i.e., nodes $k$ and $l$ ) of that link need to be hub nodes (i.e., constraint (17)).

Constraint (18) also ensures that at most one of $U_{m n}^{k l}$ and $U_{n m}^{k l}$ variables can be 1 because they need to provide a simple directed path. Constraints (19)-(21) are the flow balance constraints in the hub network. According to these constraints, every hub node sends and receives one unit of flow, and the connectivity in the hub network is established. By constraint (19), if both nodes $m$ and $l$ are hubs, then the origin hub $m$ sends one unit of flow to the destination hub $l$ in the hub network. By constraint (21), if nodes $m$ and $k$ are both hub nodes, then the destination hub $m$ receives one unit of flow from the origin hub $k$ in the hub network. If hub node $m$ is not the origin or the destination, then the incoming flow must equal the outgoing flow by constraint (20). Constraints (22) and (23) are domain constraints.

\section{Robust SRHLP}

In real settings, after designing a hub network, some parameters (e.g., demand, distance, time, and cost) in the problem may change due to uncertainty. For example, due to variability in the transportation time from an origin to a destination, there is a possibility that the flow might not be delivered on time. A failure in on-time delivery may have a huge and non-measureable cost (e.g., lost-opportunity cost and lost-sale cost due to customer churning) due to the compensation of an unsatisfied customer. Nevertheless, most proposed HLP models treat data as known and deterministic. Hence, many researchers have attempted to model the uncertainty in the design of optimization problems in recent studies [25-34].

One way of handling uncertainty is to estimate the probability distribution of the parameters, but various sources can lead to parameter uncertainty, and thus collecting historical data can be difficult and the probabilities are difficult to estimate. Similar to Peng et al. [2], we employ robust optimization so there is no need to estimate probabilistic information. The proposed approach is a form of scenario-based robust optimization, which delivers solutions with reasonable objective values under any scenario specified by the decision maker. Many previous studies in this area, particularly facility location problems, have considered various robustness criteria (see Snyder [5] for further details), where the most commonly applied robustness criteria are the minimax cost and minimax regret, which minimize the maximum cost or regret among all scenarios. One of the most efficient methods for robust optimization is the $p$-robustness measure, where the relative regret in each scenario must be no more than a constant $p$ [35]. This method has also been applied to two well-known problems to minimize the expected total cost, i.e., the $p$-robust $k$-median and $p$-robust uncapacitated fixed-charge location problems by Snyder and Daskin [13].

In the following, we apply the $p$-robustness measure in the proposed SRHLP and we refer to this method as the p-robustness SRHLP ( $p$-SRHLP). The objective function of the $p$-SRHLP model is to minimize the total expected cost while restricting the relative regret in each scenario to no more than $p$ for a constant $p>0$. A scenario-based modeling approach is used, where specific values for the parameters are considered in each scenario. To the best of our knowledge, this is the first use of the $p$-robustness measure to design a reliable hub location model in order to ensure high-level performance during disruption. First, we introduce the uncertain parameters and decision variables, which have different values in each scenario. An index $\omega$ to represent a scenario is added to the previous variables given in Section 3.3. In addition, random parameters are rewritten by adding the index $\omega$ to each of them.

Set

$\omega=\{1, \ldots, \Psi\}$ set of scenarios

\section{Parameters}

$\begin{array}{ll}W_{i j}^{\omega} & \text { flow to be sent from node } i \text { to node } j \text { in scenario } \omega . \\ C_{i k}^{\omega} & \text { transportation cost of a unit of flow from node } i \text { to node } j \text { in scenario } \omega . \\ F_{k}^{\omega} & \text { fixed cost of opening a hub at node } k \text { in scenario } \omega . \\ \eta_{k}^{\omega} & \text { capacity exceedance probability at hub } k \text { in scenario } \omega . \\ \theta_{k}^{\omega} & \text { capacity disruption factor at hub } k \text { in scenario } \omega . \\ \Phi_{\omega} & \text { probability of scenario } \omega . \\ \zeta_{\omega}^{*} & \text { optimal objective value of the model under the data from scenario } \omega\end{array}$




$$
\begin{array}{ll}
p & \text { desired robustness level, } p>0 \\
O_{i}^{\omega}=\sum_{j \in I} W_{i j}^{\omega} & \text { total flow originating at node } i \text { in scenario } \omega . \\
D_{i}^{\omega}=\sum_{j \in I} W_{j i}^{\omega} & \text { total flow destined for node } i \text { in scenario } \omega . \\
q_{k}^{\omega} & \text { probability of failure for hub } k \text { in scenario } \omega .
\end{array}
$$

Variables
$X_{i k}^{r \omega}$
Pr $\omega$
is 1 if node $i$ is allocated to hub $k$ at level $r$ in scenario $\omega$; 0 , otherwise
$P_{i k}^{r \omega}$ probability that hub $k$ serves node $i$ at level $r$ in scenario $\omega$.
$\mathrm{OFV}_{p} \quad$ objective function value for a $p$-robust model

\subsection{Definition of p-robustness}

In this section, the definition of "p-robust" given by Snyder and Daskin [13] is stated as follows.

Definition. Given a set of scenarios $(\Psi)$, consider $\mathbb{D}_{\omega}$ as the deterministic minimization problem for scenario $\omega$ (i.e., a deterministic HLP) and its optimal objective function value is shown as $\zeta_{\omega} *$. In addition, let $\boldsymbol{Z}$ and $\boldsymbol{X}$ be feasible vectors of the location and allocation variables, respectively, $\boldsymbol{U}$ and $\boldsymbol{L}$ are feasible vectors of the tree structure variables, $\boldsymbol{P}$ is the feasible vector of the probability variables, and $\zeta_{\omega}(\boldsymbol{X}, \boldsymbol{Z}, \boldsymbol{U}, \boldsymbol{L}, \boldsymbol{P})$ are the values of the objective function for the variable set $(\boldsymbol{X}, \boldsymbol{Z}, \boldsymbol{U}, \boldsymbol{L}, \boldsymbol{P})$ in scenario $\omega$. Then, $(\boldsymbol{X}, \boldsymbol{Z}, \boldsymbol{U}, \boldsymbol{L}, \boldsymbol{P})$ is called $p$-robust if for all $\omega \in \Psi$,

$$
\frac{\zeta_{\omega}(\boldsymbol{X}, \boldsymbol{Z}, \boldsymbol{U}, \boldsymbol{L}, \boldsymbol{P})-\zeta_{\omega}{ }^{*}}{\zeta_{\omega}{ }^{*}} \leq p \quad \forall \omega \in \Psi .
$$

Equivalently, constraint (24) can be stated by:

$$
\zeta_{\omega}(\boldsymbol{X}, \boldsymbol{Y}, \boldsymbol{Z}, \boldsymbol{U}, \boldsymbol{L}, \boldsymbol{P}) \leq(1+p) \zeta_{\omega}{ }^{*} \quad \forall \omega \in \Psi,
$$

where $p \geq 0$ is a given constant, which indicates the desired robustness level. The left-hand side of constraint (25) is the relative regret of the objective function in scenario $\omega$. The $p$-robust measure sets upper bounds on the maximum allowable relative regret for the objective function in each scenario. Finally, the $p$-robustness measure can be combined with the original problem to obtain a model in the following form.

$$
\min \sum_{\omega \in \Psi} \Phi_{\omega} \zeta_{\omega},
$$

s.t.

$$
\zeta_{\omega}(\boldsymbol{X}, \boldsymbol{Z}, \boldsymbol{U}, \boldsymbol{L}, \boldsymbol{P}) \leq(1+p) \zeta_{\omega}{ }^{*} \quad \forall \omega \in \Psi,
$$

$$
g(\boldsymbol{X}, \boldsymbol{Z}, \boldsymbol{U}, \boldsymbol{L}, \boldsymbol{P}) \leq(=\text { or } \geq) b .
$$

\subsection{Formulation of the $\mathrm{p}-\mathrm{SRHLP}$}

We propose the following mixed-integer programming model for the $p$-SRHLP.

$$
\begin{aligned}
\min \mathrm{OFV}_{p}= & \sum_{\omega=1}^{\Psi} \Phi_{\omega}\left\{\sum_{k=1}^{K} F_{k}^{\omega} Z_{k}+\sum_{i=1}^{I} \sum_{k=1}^{K+1} \sum_{r=1}^{R} \delta C_{i k}^{\omega}\left(O_{i}^{\omega}+D_{i}^{\omega}\right) P_{i k}^{r \omega} X_{i k}^{r \omega}\right. \\
& \left.+\sum_{m=1}^{K+1} \sum_{n=1}^{K+1} \alpha C_{m n}^{\omega}\left[\sum_{i=1}^{I} \sum_{\substack{j=1 \\
j \neq i}}^{I} \sum_{k=1}^{I} \sum_{\substack{l=1 \\
l \neq k}}^{K+1} \sum_{r=1}^{R} \sum_{s=1}^{R} W_{i j}^{\omega} U_{m n}^{k l} r_{i m}^{r \omega} X_{i m}^{r \omega} P_{j n}^{s \omega} X_{j n}^{s \omega}\right]\right\},
\end{aligned}
$$

s.t.

$$
\begin{aligned}
& \sum_{k=1}^{K} F_{k}^{\omega} Z_{k}+\sum_{i=1}^{I} \sum_{k=1}^{K+1} \sum_{r=1}^{R} \delta C_{i k}^{\omega}\left(O_{i}^{\omega}+D_{i}^{\omega}\right) P_{i k}^{r \omega} X_{i k}^{r \omega} \\
& \quad+\sum_{m=1}^{K+1} \sum_{n=1}^{K+1} \alpha C_{m n}^{\omega}\left[\sum_{i=1}^{I} \sum_{\substack{j=1 \\
j \neq i}}^{I} \sum_{k=1}^{I} \sum_{\substack{l=1 \\
l \neq k}}^{K+1} \sum_{r=1}^{R} \sum_{s=1}^{R} W_{i j}^{\omega} U_{m n}^{k l} P_{i m}^{r \omega} X_{i m}^{r \omega} P_{j n}^{s \omega} X_{j n}^{S \omega}\right] \leq(1+p) \zeta_{\omega} * \quad \forall \omega \in \Psi,
\end{aligned}
$$




$$
\begin{aligned}
& \sum_{k=1}^{K} X_{i k}^{r \omega}+\sum_{s=1}^{r} X_{i(K+1)}^{s \omega}=1 \quad \forall i \in I, r \in\{1, \ldots, R\}, \omega \in \Psi \\
& \sum_{r=1}^{R} X_{i(K+1)}^{r \omega}=1 \quad \forall i \in I, \omega \in \Psi \\
& P_{i k}^{1 \omega}=1-q_{k}^{\omega} \quad \forall i \in I, k \in\{1, \ldots, K+1\}, \omega \in \Psi \\
& P_{i l}^{r \omega}=\left(1-q_{l}^{\omega}\right) \sum_{k=1}^{K} \frac{q_{k}^{\omega}}{1-q_{k}^{\omega}} P_{i k}^{r-1, \omega} X_{i k}^{r-1, \omega} \quad \forall i \in I, l \in\{1, \ldots, K+1\}, r \in\{2, \ldots, R\}, \omega \in \Psi, \\
& \sum_{r=1}^{R} X_{i k}^{r \omega} \leq Z_{k} \quad \forall i \in I, k \in\{1, \ldots, K\}, \omega \in \Psi \\
& \sum_{m=1}^{K+1} \sum_{i=1}^{I} \sum_{\substack{j=1 \\
j \neq i}}^{I} \sum_{k=1}^{I} \sum_{\substack{l=1 \\
l \neq k}}^{K+1} \sum_{r=1}^{R} \sum_{s=1}^{R} W_{i j}^{\omega} U_{m n}^{k l} P_{i m}^{r \omega} X_{i m}^{r \omega} P_{j n}^{s \omega} X_{j n}^{s \omega} \leq \bar{\Gamma}_{n}\left[\theta_{n}^{\omega}\left(1-\eta_{n}^{\omega}\right)+\eta_{n}^{\omega}\right] \\
& \forall n \in\{1, \ldots, K\}, \omega \in \Psi \\
& X_{i k}^{r \omega}, Z_{k}, U_{m n}^{k l}, L_{k l} \in\{0,1\} \quad \forall i \in I ; k, m, n \in\{1, \ldots, K+1\} ; r \in R, \omega \in \Psi, \\
& P_{i k}^{r \omega} \geq 0 \quad \forall i \in I ; \quad k, l \in\{1, \ldots, K+1\} ; r \in R, \omega \in \Psi
\end{aligned}
$$

(12),(13), (16)-(21)

\subsection{Identifying disruption scenarios}

An important issue is how to determine the disruption scenarios. For a given set of $S$ possible scenarios, there are a total of $2^{S}$ possible disruption scenarios, which leads to exponential growth of the problem size and a huge computational time is required to solve the problem. In addition, if there are a large number of scenarios, carefully determining $p$ is highly important for maintaining the feasibility.

The number of scenarios is much smaller in real settings, so decision makers may determine scenarios based on the preferences of experts. One method to determine a set of scenarios may involve a situation where exactly one hub node is disrupted (i.e., complete or partial disruptions). This set of scenarios is determined by considering the hubs that are more likely to be disrupted according to historical data or the a priori beliefs of decision makers [2]. In real world cases, the probability of more than two unrelated hubs being disrupted at the same time is very small, so this set of scenarios is neglected in this study. Therefore, in the case of multiple hub disruption, the hubs located in the same area have a higher probability of being disrupted due to certain events (e.g., earthquakes and electricity shortages) [2].

In the case of a large number of scenarios, different sets of scenarios may be determined approximately using sample average approximation [36], where each scenario can be generated using the Monte Carlo simulation method.

\section{Lower bound and meta-heuristic algorithm}

The model presented in Section 3.3 is a mixed-integer nonlinear programming model. Several optimization techniques have been ever developed to solve this model (e.g., branch-and-bound, branch-and-cut, and branch-and-price). However, these techniques can be computationally demanding, especially when the number of scenarios is large, but decision makers are often more interested in obtaining near-optimal solutions in a relatively short computational time.

The proposed model was coded in GAMS 22.9 but solving large size instances (up to 15 nodes) required a computational time of several weeks. Due to this limitation, we developed an efficient meta-heuristic algorithm called the self-adaptive imperialist competitive algorithm (SAICA) to find a sufficiently good (i.e., near optimal) solution in a reasonable amount of computational time, especially for real sized problems. Furthermore, we compared the performance of the proposed SAICA with an efficient lower bound method based on [27]. In the following, the proposed SAICA is explained for the given problem. First, a tailored solution representation devised for the problem is illustrated and the details of the SAICA are then elaborated. Finally, in order to evaluate the performance of the meta-heuristic algorithm, a lower bound is developed based on the approach of Mohammadi et al. [27]. 


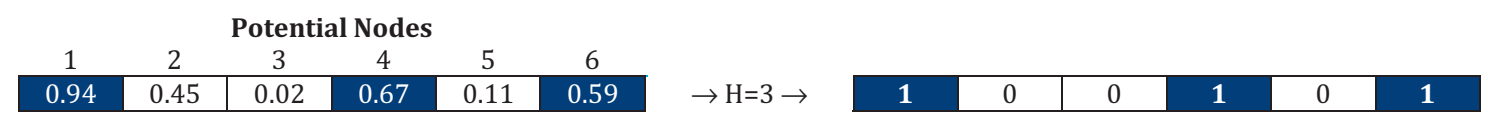

Fig. 2. Hub location scheme.

\begin{tabular}{|c|c|c|c|c|c|c|c|c|}
\hline \multicolumn{9}{|c|}{ Potential Hub Nodes } \\
\hline & & 1 & 2 & 3 & 4 & 5 & 6 & Non-failable hub \\
\hline \multicolumn{2}{|l|}{ Location of Hubs } & 1 & 0 & 0 & 1 & 0 & 1 & 1 \\
\hline Hub & 1 & 0.51 & 0 & 0 & 0.79 & 0 & 0.60 & 0.67 \\
\hline Non-hub & 2 & 0.40 & 0 & 0 & 0.85 & 0 & 0.03 & 0.75 \\
\hline Non-hub & 3 & 0.72 & 0 & 0 & 0.15 & 0 & 0.27 & 0.74 \\
\hline Hub & 4 & 0.91 & 0 & 0 & 0.03 & 0 & 0.04 & 0.39 \\
\hline Non-hub & 5 & 0.63 & 0 & 0 & 0.84 & 0 & 0.99 & 0.65 \\
\hline Hub & 6 & 0.09 & 0 & 0 & 0.93 & 0 & 0.12 & 0.17 \\
\hline Hub & 1 & - & - & - & - & - & - & - \\
\hline Non-hub & 2 & - & - & - & 1 & - & - & 2 \\
\hline Non-hub & 3 & - & - & - & - & - & - & 1 \\
\hline Hub & 4 & - & - & - & - & - & - & - \\
\hline Non-hub & 5 & - & - & - & 2 & - & 1 & 3 \\
\hline Hub & 6 & - & - & - & - & - & - & - \\
\hline
\end{tabular}

Fig. 3. Allocation scheme.

\subsection{Solution representation}

An encoded HLP solution should show the locations of the hub nodes and the allocations of the origin and destination nodes for the located hubs. In this study, a continuous solution representation (CSR) is developed for the HLP, which avoids generating infeasible solutions during the search process and the solution process is made much easier. The proposed CSR comprises three components: (1) locations of the hubs, (2) allocations of non-hub nodes to the located hub, and (3) structure of the hub network, which are explained in detail as follows.

\subsubsection{CSR for the location of hubs}

The first matrix corresponds to the location decision represented by a $(1 \times \boldsymbol{K})$ matrix (i.e., location matrix), where $\boldsymbol{K}$ denotes the number of potential hub nodes. This matrix is filled with random numbers from $[0,1]$. In this matrix, the first maximum $\boldsymbol{H}$ numbers are considered to be located hubs, as shown in Fig. 2. In this figure, three hubs must be located among six nodes; therefore, the first, fourth, and sixth nodes are located as hubs.

\subsubsection{CSR for the allocation of non-hub nodes to the located hub}

This section represents the allocation of non-hub nodes to the located hubs. The non-hub nodes are allocated to the failable hubs level by level after they have been allocated to the dummy non-failable hub at a certain level. This part of the solution contains the matrix $(\boldsymbol{I} \times \boldsymbol{K}+1)$, which is filled with random numbers from $[0,1]$. First, each row of the matrix is multiplied by the location matrix. Next, each non-hub node is allocated to its biggest corresponding non-zero column as the first level of allocation and this value is replaced by 1 . Moreover, the non-hob node is allocated to the next big value for the next level of allocation and this value is replaced by 2 . This procedure is repeated $R$ times (i.e., the maximum number of allocation levels) after the non-hub node has been allocated to the dummy non-failable hub in the last column. If the non-hub has not been allocated to the dummy non-failable hub at $(R-1)$ th level, then that non-hub node is allocated intentionally to the dummy non-failable hub at level $R$. Thus, the last column of that non-hub node is replaced by $R$. Fig. 3 shows the non-hub allocation for the previous example with $R=3$.

For example, in Fig. 3, non-hub number 2 is allocated to failable hub number 4 at the first level and allocated to the dummy non-failable hub at the second level. Furthermore, non-hub number 3 is only allocated to the dummy non-failable hub at level one. Moreover, non-hub number 5 is allocated to failable hubs numbers 6 and 4 and the dummy non-failable hub for the first, second, and third levels of allocation, respectively.

\subsubsection{CSR for the structure of the hub network}

This part of the solution representation depicts the structure of the hub network. The network of hubs is a tree structure, so a Prüfer sequence method is used [37]. The Prüfer sequence of a labeled tree is a unique sequence associated with the tree. The sequence for a tree on $n$ vertices has a length of $n-2$ and it can be generated by a simple iterative algorithm. 


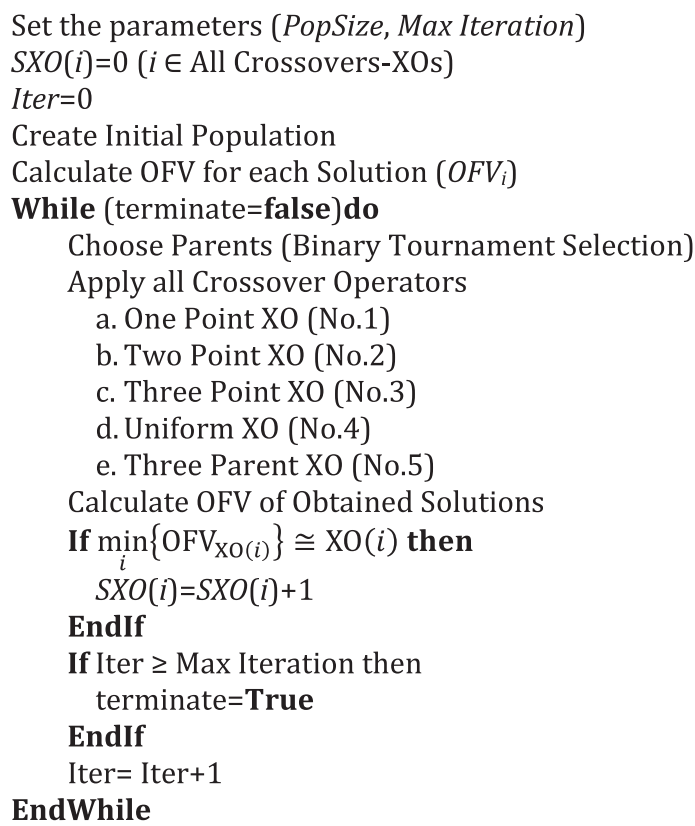

Calculate Selection Probabilty $\left(S P(i)=\frac{S X O(i)}{\sum_{i} S X O(i)}\right)$

Fig. 4. Pseudo code of the SAICA's Initialization phase.

\subsection{Proposed SAICA}

Similar to most other evolutionary algorithms such as genetic algorithms [38,39] and particle swarm optimization, the ICA is a population-based algorithm [40,41] and it was introduced by Atashpaz-Gargari and Lucas [42]. Thus, the ICA begins with an initial population of random solutions, where each member of the population is called a country (see Mohammadi et al. [26] for further details). In this study, we propose a self-adaptive variant of the ICA called SAICA, which obtains higher quality solutions compared with pure ICA. In the proposed SAICA, a self-adaptive crossover operator is applied in addition to the original assimilation and revolution operators in ICA. Various crossover operators (e.g., one point, two points, uniform, and cycle crossover) have been introduced previously, but employing all of them simultaneously in a single algorithm would increase the computational time tremendously. Therefore, many studies have used only one to search the solution space. By contrast in the proposed SAICA, several crossover operators are applied simultaneously without increasing the computational time.

The SAICA contains initialization and main phases. In the initialization phase, each crossover operator is rewarded with a score after comparing it with other operators if it can generate a better solution at each iteration. After ending the initialization phase following a predefined number of iterations (i.e., Max Iteration), the selection probability (SP) metric is calculated for each operator by dividing the score obtained by the number of iterations, where $\sum^{S} P=1$. After the initialization phase, the main phase is started, which includes searching the solution space using the assimilation, self-adapted crossover, and revolution operators. In this study, the crossover operators comprise one point, two points, three points, uniform, and three parent crossover operators [43]. The pseudo-codes for the two phases of the proposed SAICA are shown separately in Figs. 4 and 5. In order to understand the proposed SAICA more easily, flowcharts of the initialization and main phases are depicted in Figs. 6 and 7.

\subsection{Lower bound procedure}

In order to evaluate the effectiveness of the proposed meta-heuristic algorithm, a new lower bound procedure is presented in this section. As shown in the proposed model, non-hub nodes are reallocated to other hubs at a higher level when any lower hub fails to provide services. If all of the hubs fail for a non-hub node, there is a dummy hub denoted by $K+1$, which has no fixed cost with a failure probability $q_{K+1}=0$ and a transportation $\operatorname{cost} c_{i, K+1}^{1}=\phi_{i}$, for non-hub node $i$.

Let $q_{[1]}^{\omega} \leq q_{[2]}^{\omega} \leq \ldots \leq q_{[l-1]}^{\omega} \leq q_{[l]}^{\omega}$ be an ordering of the failure probabilities in $K$ for scenario $\omega$. An optimistic version of $P_{i k}^{r \omega}$ can be provided as $\mathcal{L}_{k r}^{\omega}$, which is calculated using Eq. (39) and it needs to be proved that $\mathcal{L}_{k r}^{\omega}$ is a lower bound for $P_{i k}^{r \omega}$, 
$N F C=0$

Set the parameters $\left(N_{P o p}, N_{i m p}, \xi, \beta, \alpha, P_{A}, P_{C}, P_{R}\right)$

Generate initial Countries containing all distinct five parts $\left(X_{i} \mid i=1, \ldots, N_{\text {pop }}\right)$

Evaluate fitness of each country $\left(O F V_{i}\right)$

Form initial empires:

Choose first $N_{\text {imp }}$ countries as the empires $\min _{i}\left\{O F V_{i} \mid i=1, \ldots, N_{\text {Pop }}\right\}$

$N_{\text {Colony }}=N_{\text {Pop }}-N_{\text {imp }}$

For $i=1: N_{\text {imp }}$

$$
P_{\text {empire }_{i}}=e^{\left(-\alpha \frac{\text { oFV }_{\text {empire }_{i}}}{\max _{k}\left\{\text { oFV }_{\text {empire } \left._{k}\right\}}\right\}}\right)}
$$

\section{EndFor}

$N P_{\text {empire }_{i}}=\frac{P_{\text {empire }_{i}}}{\sum_{k=1}^{N_{\text {imp }} P_{\text {empire }_{k}}}}(N P:$ Normalized Probability $)$

$N C_{\text {empire }_{k}}=N P_{\text {empire }_{k}} \times N_{\text {Colony }}(N C$ : Number of Colony)

Assign colonies to their related empire ( $\left.\mathrm{pop}_{1}\right)$ as:

For $i=1: N_{\text {Colony }}$

If $\mathbf{C}_{-} \mathbf{N P}_{\mathbf{P}_{\mathbf{e}_{\text {empire }} \mathbf{k}-\mathbf{1}}} \leq \operatorname{rand}() \leq \mathbf{C}_{-} \mathbf{N P}_{\mathbf{P}_{\text {empire }_{\mathbf{k}}}}$ then $\left(C_{-} N P\right.$ : Cumulative Normalized Probability $)$

\section{EndIf}

Assign $i$ th Colony to $k$ th Empire

\section{EndFor}

While (terminate $=$ false) do at each Imperialist

Assimilate colonies to their related empire as: $\left(\mathrm{pop}_{2}\right) \leftarrow P_{A}, \beta$

$$
X_{\text {colony }}^{\text {New }}=X_{\text {colony }}^{\text {old }}+\beta \cdot \operatorname{rand}() \cdot\left|X_{\text {colony }}^{\text {old }}-X_{\text {empire }}\right|
$$

Apply roulette wheel selection to choose crossover operator

Apply crossover operator based on Initialization phase as: $\left(\mathrm{pop}_{3}\right) \leftarrow P_{C}, S P(i)$

If $C_{-} S P_{\mathrm{XO}(i)-1} \leq \operatorname{rand}() \leq C_{-} S P_{\mathrm{XO}(i)}$ then

\section{EndIf}

Apply XO $(i)$ on the parents

Perform Revolution among colonies $\left(\mathrm{pop}_{4}\right) \leftarrow P_{R}$

Evaluate fitness of new created solutions $\left(O F V_{i}\right)$

$\mathrm{Pop}_{\text {New }}=\left\{\mathrm{Pop}_{1} \cup \mathrm{Pop}_{2} \cup \mathrm{Pop}_{3} \cup \mathrm{Pop}_{4}\right\}$

If $(O F V$ colony < OFVempire $)$ then

$$
\begin{aligned}
& X_{\text {empire }}^{\text {New }}=X_{\text {colony }}^{\text {New }} \\
& X_{\text {colony }}^{\text {New }}=X_{\text {empire }}^{\text {Old }}
\end{aligned}
$$

\section{EndIf}

Calculate total power of the imperialists $\left(T P I_{k}\right)$ as: $\longleftarrow \xi$

Perform imperialistic competition

$$
T P I_{k}=O F V \text { empire }+\xi . \frac{\sum_{i=1}^{N C_{\text {empire }_{k}}} \text { OFV }_{\text {colony }_{i}}^{\text {empire }_{k}}}{N C_{\text {empire }_{k}}}
$$

Eliminate the powerless empires (imperialist with no colony)

If $(\mathrm{NFC}=$ predefined value) then

Terminate $=$ true

\section{EndIf}

EndWhile

Fig. 5. Pseudo code of the SAICA's main phase.

as proved previously in [44].

$$
\mathcal{L}_{k r}^{\omega}= \begin{cases}\left(\prod_{l=1}^{r} q_{[l]}^{\omega}\right)\left(1-q_{k}\right) & k \in K, \\ \prod_{l=1}^{r-1} q_{[l]} & k=K+1 .\end{cases}
$$

According to Eq. (39), the approximated SRHLP can be represented as the following model, $\mathbb{P}$. 


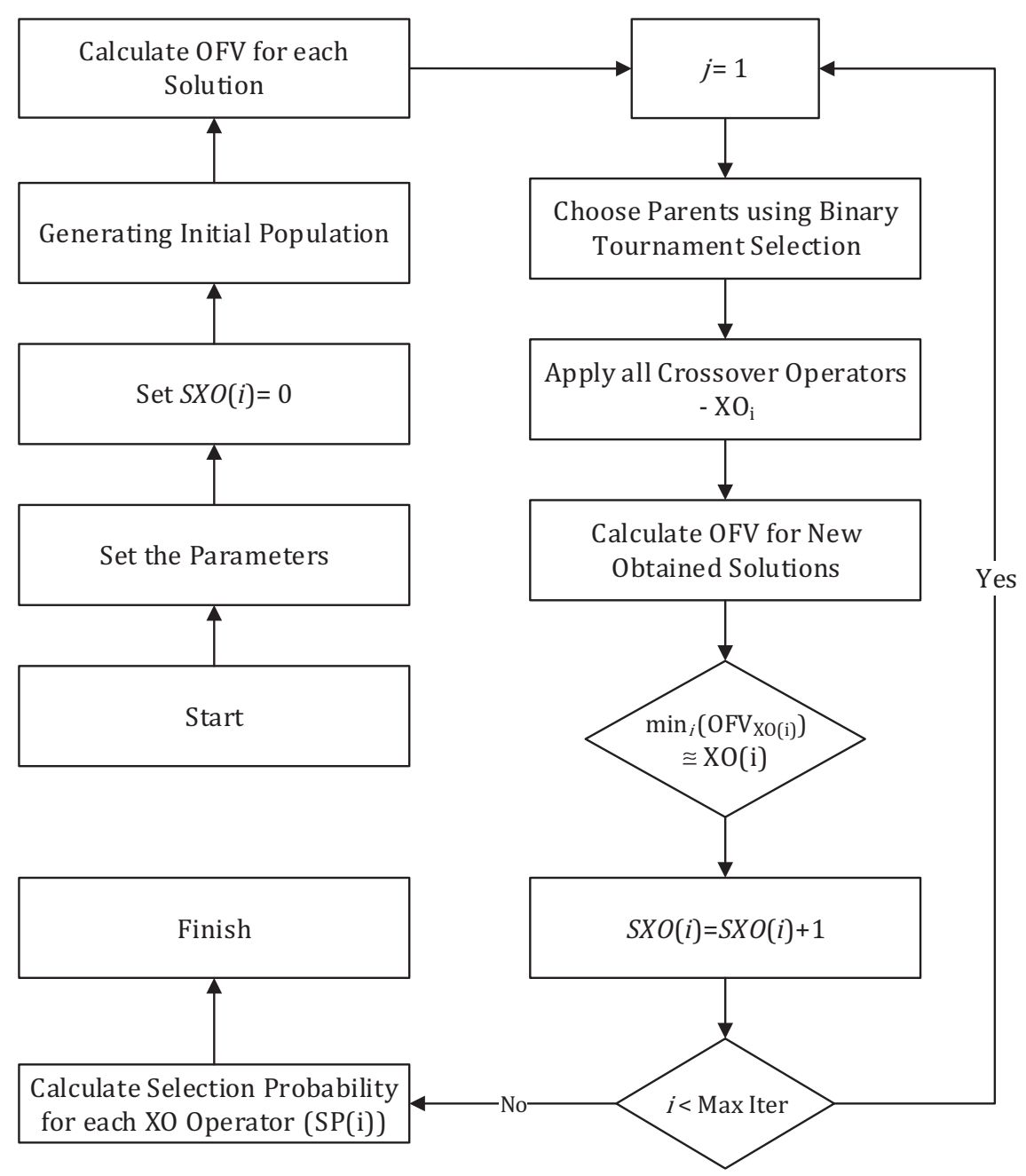

Fig. 6. Flowchart of the initialization phase.

Approximated model $\mathbb{P}$ :

$$
\begin{aligned}
& \min \sum_{\omega=1}^{\Psi} \Phi_{\omega}\left\{\sum_{k=1}^{K} F_{k}^{\omega} Z_{k}+\sum_{i=1}^{I} \sum_{k=1}^{K+1} \sum_{r=1}^{R} \delta C_{i k}^{\omega}\left(O_{i}^{\omega}+D_{i}^{\omega}\right) \mathcal{L}_{k r}^{\omega} X_{i k}^{r \omega}\right. \\
& \left.+\sum_{m=1}^{K+1} \sum_{n=1}^{K+1} \alpha C_{m n}^{\omega}\left[\sum_{i=1}^{I} \sum_{\substack{j=1 \\
j \neq i}}^{I} \sum_{l=1}^{I} \sum_{\substack{k=1 \\
k \neq l}}^{K+1} \sum_{r=1}^{R} \sum_{s=1}^{R} W_{i j}^{\omega} U_{m n}^{k l} \mathcal{L}_{m r}^{\omega} X_{i m}^{r \omega} \mathcal{L}_{n s}^{\omega} X_{j n}^{s \omega}\right]\right\},
\end{aligned}
$$

s.t.

$$
\begin{aligned}
\sum_{k=1}^{K} F_{k}^{\omega} Z_{k}+\sum_{i=1}^{I} \sum_{k=1}^{K+1} \sum_{r=1}^{R} \delta C_{i k}^{\omega}\left(O_{i}^{\omega}+D_{i}^{\omega}\right) \mathcal{L}_{k r}^{\omega} X_{i k}^{r \omega} \\
+\sum_{m=1}^{K+1} \sum_{n=1}^{K+1} \alpha C_{m n}^{\omega}\left[\sum_{i=1}^{I} \sum_{\substack{j=1 \\
j \neq i}}^{I} \sum_{l=1}^{I} \sum_{\substack{k=1 \\
k \neq l}}^{K+1} \sum_{r=1}^{R} \sum_{s=1}^{R} W_{i j}^{\omega} U_{m n}^{k l} \mathcal{L}_{m r}^{\omega} X_{i m}^{r \omega} \mathcal{L}_{n s}^{\omega} X_{j n}^{s \omega}\right] \leq(1+p) \zeta_{\omega} * \forall \omega \in \Psi
\end{aligned}
$$




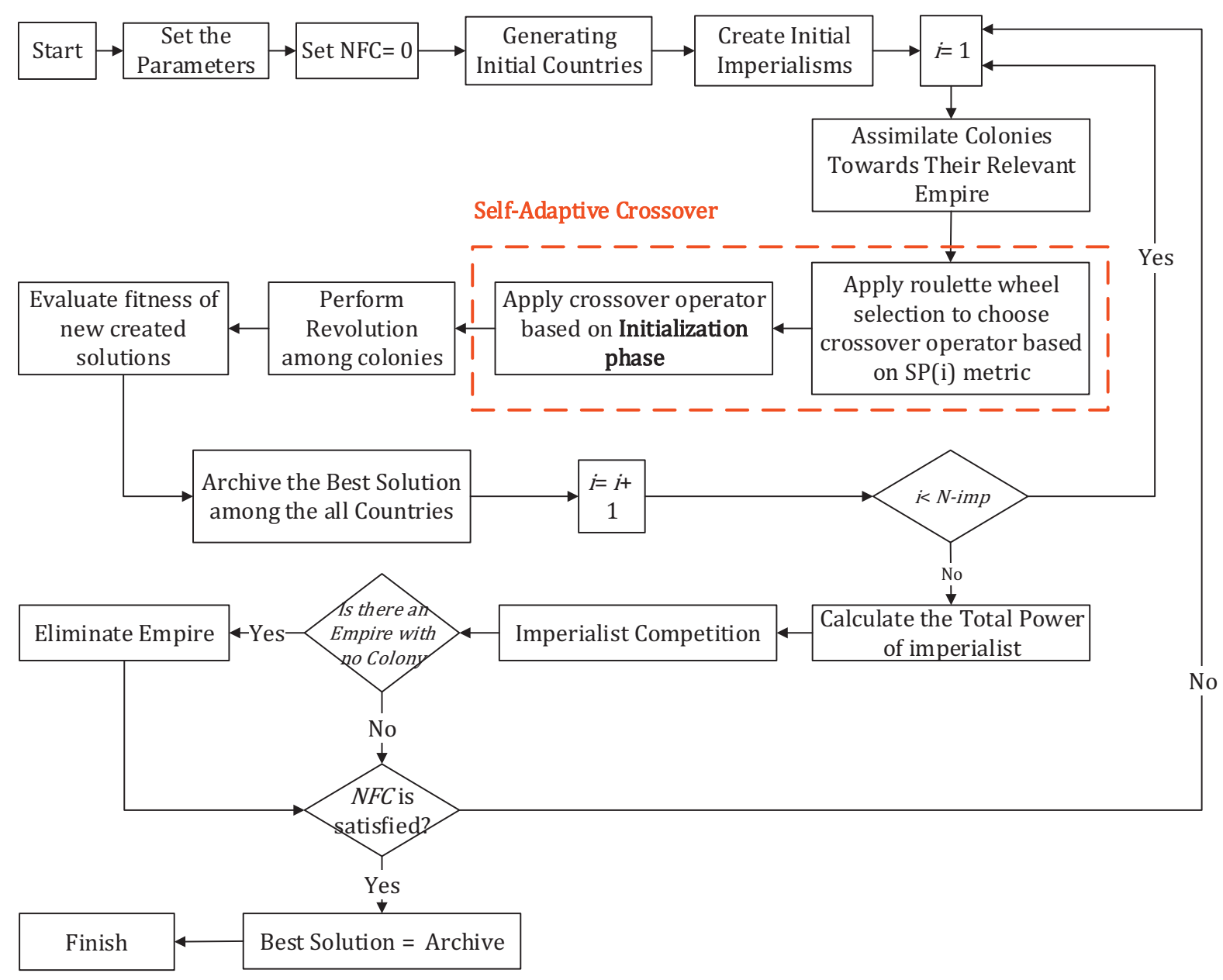

Fig. 7. Flowchart of the main phase.

$$
\sum_{m=1}^{K+1} \sum_{i=1}^{I} \sum_{\substack{j=1 \\ j \neq i}}^{I} \sum_{l=1}^{I} \sum_{\substack{k=1 \\ k \neq l}}^{K+1} \sum_{r=1}^{R} \sum_{s=1}^{R} W_{i j}^{\omega} U_{m n}^{k l} \mathcal{L}_{m r}^{\omega} X_{i m}^{r \omega} \mathcal{L}_{n s}^{\omega} X_{j n}^{s \omega} \leq \bar{\Gamma}_{n}\left[\theta_{n}^{\omega}\left(1-\eta_{n}^{\omega}\right)+\eta_{n}^{\omega}\right] \quad \forall n \in\{1, \ldots, K\}, \omega \in \Psi
$$

(12),(13), (16)-(21), (31), (32), (35), (37)

In addition to the above approximation method, according to Mohammadi et al. [27], we use a partial relaxation of a sub-problem of the approximated problem $\mathbb{P}$. In this procedure, a sub-problem of $\mathbb{P}$ can be created by dividing the set of hubs, where any two sets of hubs, $S_{g}$ and $S_{q}$, are distinct if there is at least one hub $k \in S_{g}$ and $k \notin S_{q}$. Thus, there are $C_{H}^{|K|}=K ! /((K-H) ! H !)$ distinct hub sets in a network. For a selected set of hubs $S_{g}$, where $\left|S_{g}\right|=H$, the corresponding location decision variables $Z_{k}$ are fixed at 1 and $\mathbb{P}$ is then reduced to a mixed-integer non-linear assignment sub-problem $\left(S \mathbb{P}_{g}\right)$ over the binary decision variables $X, U, L$. Now, let $R \mathbb{P}\left(S \mathbb{P}_{g}\right)$ be the partial relaxation of $S \mathbb{P}_{g}$, where the integrality of the other binary variables is relaxed (i.e., $\left.0<X_{i k}^{r \omega}, L_{k l}, U_{m n}^{k l}<1\right)$. Moreover, let $O F V_{R \mathbb{P}(S \mathbb{P} g}$ be the optimal objective function value of $R \mathbb{P}\left(S \mathbb{P}_{g}\right)$. This value can be declared as a lower bound for the optimal value of $S \mathbb{P}$, so the minimum value among these values from the possible $C_{H}^{|K|}$ sets determines a lower bound for the optimal value of the original problem $\mathbb{P}$, where $\mathrm{OFV}_{L B(\mathbb{P})}=\min _{g} \mathrm{OFV}_{R \mathbb{P}(S \mathbb{P} g}$.

\section{Computational results}

We performed a number of numerical experiments to evaluate the performance of the proposed algorithm. We encoded the algorithm in $\mathrm{C}++$ and executed it on a Pentium 4 computer with a $3.0 \mathrm{GHz}$ CPU and 16 GB RAM, which operated Mi- 
Table 1

Randomly generated parameters.

\begin{tabular}{lllllll}
\hline & Parameters & & & \\
\cline { 2 - 6 } & $W$ & $C$ & $F$ & $\eta$ & $\theta$ & $\Gamma$ \\
\hline Values & $\sim \mathrm{U}(20,500)$ & $\sim \mathrm{U}(50,1500)$ & $\sim \mathrm{U}(5 \mathrm{e} 3,2 \mathrm{e} 4)$ & $\sim \mathrm{U}(0,0.3)$ & $\sim \mathrm{U}(0.5,1)$ & $\sim \mathrm{U}(1 \mathrm{e} 3,5 \mathrm{e} 3)$ \\
\hline
\end{tabular}

Table 2

SAICA parameters settings.

\begin{tabular}{llll}
\hline Parameters & Settings & Parameters & Settings \\
\hline Population size & 200 & $\xi$ & 0.05 \\
Imperialist no. & 8 & $\beta$ & 1.8 \\
Assimilation rate & 0.80 & NFC & 50,000 \\
Revolution rate & 0.20 & & \\
\hline
\end{tabular}

crosoft Windows 7 Ultimate. We benchmarked our results using the nonlinear branch and reduced the approach in BARON solver. The computational times are reported in seconds.

\subsection{Experimental design}

We generated 45 random test problems with different sizes in terms of the number of nodes $(I)$, number of hubs $(H)$, number of assignment levels $(R)$, and number of scenarios $(\Psi)$, which ranged from 10 to 200,4 to 25,2 to 5 , and 10 to 50 , respectively. The test problems were labeled as " $I-H-R-\Psi$ ". For example, instance " $40-8-4-15$ " had 40 non-hub nodes, eight hub nodes, four assignment levels, and 15 scenarios. For all of the test problems, it was considered that all nonhub nodes could be origin and destination nodes at the same time. Disruption scenarios were generated randomly where each hub could be disrupted with a probability of $q_{k}^{\omega}=0.15$. This probability was intentionally selected as high in order to determine the impact of disruptions and to assess the performance of the model when disruptions were a significant factor. For duplicate scenarios, a new scenario was generated and the procedure was repeated until $\Psi$ unique scenarios were generated.

The feasibility of the model depends strongly on the value of $p$. For instance, $p=1$ will usually guarantee the feasibility of the model, but it is less realistic because it allows a large increase in the cost of disrupted scenarios. By contrast, small values of $p$ lead to infeasibility. Heuristic algorithms are available to find the minimum value of $p$ in order to obtain feasible solutions and a method for finding the minimum feasible $p$ in the proposed SRHLP is introduced in Section 6.4. We performed all of the experiments with the same value of $p=0.15$ for consistency. The other parameters were generated uniformly as shown in Table 1 .

\subsection{Performance of SAICA}

In this section, we present comparisons between the performance of the proposed SAICA and the lower bound procedure (LB). First, the required parameters in the proposed SAICA algorithm were tuned using the well-known response surface methodology (RSM) and they are tabulated in Table 2. Due to space limitations, the details of the RSM results are not presented but they can be provided on request. The parameter settings were pre-tested over randomly selected problem instances before they were used in the comparative study. All evolutionary algorithms are stochastic in nature and their performance often fluctuates, so for all 45 test problems, each instance was generated 20 times and the average value of the results is presented. The results of the performance comparison are shown in Table 3. The "Test Problem" column shows the instance name. The columns "Time" and "OFV" present the run time and the objective value for both the LB and SAICA.

First, in order to determine the accuracy of the proposed $\mathrm{LB}$, the difference between $\mathbf{O F V}_{\mathrm{LB}(\mathbb{P})}$ and the optimal solution was calculated for 15 small test problems as: $\left[100 \times\left(\mathbf{O F V} \mathbf{V}_{p}-\mathbf{O F V}_{\mathrm{LB}(\mathbb{P})}\right) / \mathbf{O F V} \mathbf{L B}_{\mathrm{LB}(\mathbb{P})}\right]$. According to Fig. 8, the mean difference between the LB and optimal solution was $0.48 \%$. Therefore, the accuracy of the LB was about $0.48 \%$ in comparison with optimal solutions.

The LB method and SAICA are compared in the "\% GAP" column, where the "Time" column shows the percentage of CPU time under the LB required by the proposed SAICA algorithm and "OFV" indicates the difference (as a percentage) in the objective function values. A value less than $100 \%$ in the "Time" column indicates the better performance of the proposed SAICA in terms of the CPU time, which was the case for all of the test problems. According to the "OFV" column as "\% GAP," the mean difference between the proposed SAICA and LB procedure was $0.89 \%$. The mean difference between the LB and optimal solution was $0.48 \%$, so the mean difference between the proposed SAICA and the optimal solution was $0.41 \%$ (i.e., $0.89-0.48 \%)$.

As shown in Table 3, compared with the LB, our proposed algorithm found near-optimal solutions for all of data sets with a mean difference of $0.89 \%$, but it required only a small fraction of the time taken by LB (18.76\% on average). It should also be noted that the CPU time generally increased with the number of scenarios in most cases. 
Table 3

SAICA performance - vs. LB.

\begin{tabular}{|c|c|c|c|c|c|c|}
\hline \multirow[t]{2}{*}{ Test problem } & \multicolumn{2}{|l|}{ LB } & \multicolumn{2}{|c|}{ SAICA } & \multicolumn{2}{|l|}{$\%$ GAP } \\
\hline & Time & OFV & Time & OFV & Time (\%) & OFV (\%) \\
\hline $10-3-2-10$ & 18 & 286,572 & 13 & 288,119 & 72.22 & 100.54 \\
\hline $10-4-2-10$ & 19 & 291,881 & 14 & 293,486 & 73.68 & 100.55 \\
\hline $15-3-2-12$ & 26 & 315,627 & 19 & 317,299 & 73.07 & 100.53 \\
\hline $15-4-2-16$ & 32 & 321,438 & 20 & 323,238 & 62.50 & 100.56 \\
\hline $15-5-3-14$ & 30 & 305,772 & 21 & 307,576 & 70.00 & 100.59 \\
\hline $25-3-2-16$ & 93 & 410,319 & 31 & 412,822 & 33.33 & 100.61 \\
\hline $25-4-2-16$ & 96 & 416,278 & 36 & 418,775 & 37.50 & 100.6 \\
\hline $25-5-3-18$ & 112 & 407,551 & 31 & 410,119 & 27.67 & 100.63 \\
\hline $25-6-3-18$ & 117 & 412,715 & 33 & 415,563 & 28.21 & 100.69 \\
\hline $25-7-3-10$ & 101 & 396,406 & 34 & 398,943 & 33.66 & 100.64 \\
\hline $40-5-2-10$ & 257 & 513,284 & 46 & 516,826 & 17.90 & 100.69 \\
\hline $40-6-3-10$ & 263 & 507,426 & 49 & 511,080 & 18.63 & 100.72 \\
\hline $40-7-3-12$ & 289 & 519,320 & 48 & 523,007 & 16.61 & 100.71 \\
\hline $40-8-3-12$ & 293 & 511,815 & 51 & 515,654 & 17.41 & 100.75 \\
\hline $70-6-2-12$ & 615 & 731,492 & 79 & 736,832 & 12.84 & 100.73 \\
\hline $70-7-3-10$ & 609 & 728,391 & 77 & 734,145 & 12.64 & 100.79 \\
\hline $70-8-3-30$ & 623 & 724,555 & 81 & 730,134 & 13.00 & 100.77 \\
\hline $70-9-4-40$ & 691 & 731,581 & 82 & 737,068 & 11.86 & 100.75 \\
\hline $70-10-4-45$ & 759 & 729,290 & 84 & 734,832 & 11.07 & 100.76 \\
\hline $100-8-4-16$ & 1831 & $1,422,073$ & 138 & $1,433,165$ & 7.54 & 100.78 \\
\hline $100-10-3-16$ & 1842 & $1,410,332$ & 144 & $1,421,474$ & 7.82 & 100.79 \\
\hline $100-10-4-18$ & 1948 & $1,417,610$ & 147 & $1,428,951$ & 7.54 & 100.8 \\
\hline $100-12-4-20$ & 1944 & $1,421,672$ & 149 & $1,433,188$ & 7.66 & 100.81 \\
\hline $100-14-4-30$ & 2132 & $1,428,100$ & 153 & $1,439,668$ & 7.18 & 100.81 \\
\hline $100-16-4-45$ & 2159 & $1,431,209$ & 158 & $1,443,088$ & 7.32 & 100.83 \\
\hline $120-12-3-20$ & 2895 & $1,620,319$ & 203 & $1,634,416$ & 7.01 & 100.87 \\
\hline $120-12-4-30$ & 3214 & $1,689,372$ & 210 & $1,704,407$ & 6.53 & 100.89 \\
\hline $120-14-4-35$ & 3231 & $1,722,119$ & 208 & $1,738,479$ & 6.43 & 100.95 \\
\hline $120-14-5-40$ & 3244 & $1,673,291$ & 215 & $1,688,853$ & 6.62 & 100.93 \\
\hline $120-16-3-45$ & 2958 & $1,734,880$ & 235 & $1,751,882$ & 7.94 & 100.98 \\
\hline $120-18-4-50$ & 3031 & $1,702,315$ & 229 & $1,718,487$ & 7.55 & 100.95 \\
\hline $150-14-5-25$ & 4752 & $1,844,351$ & 378 & $1,863,717$ & 7.95 & 101.05 \\
\hline $150-16-5-25$ & 4763 & $1,851,618$ & 383 & $1,871,615$ & 8.04 & 101.08 \\
\hline $150-18-4-30$ & 4979 & $1,923,511$ & 392 & $1,944,862$ & 7.87 & 101.11 \\
\hline $150-18-5-35$ & 5022 & $2,021,632$ & 389 & $2,042,657$ & 7.75 & 101.04 \\
\hline $150-20-4-40$ & 5104 & $2,104,371$ & 392 & $2,129,203$ & 7.68 & 101.18 \\
\hline $150-20-5-45$ & 5149 & $1,978,439$ & 391 & $2,000,795$ & 7.59 & 101.13 \\
\hline $150-20-6-50$ & 5348 & $2,112,412$ & 403 & $2,136,916$ & 7.53 & 101.16 \\
\hline $200-16-3-35$ & 7482 & $2,648,528$ & 638 & $2,683,489$ & 8.52 & 101.32 \\
\hline $200-16-4-35$ & 7495 & $2,521,339$ & 629 & $2,553,864$ & 8.39 & 101.29 \\
\hline $200-18-4-40$ & 7745 & $2,768,341$ & 641 & $2,804,329$ & 8.28 & 101.3 \\
\hline $200-18-5-40$ & 7753 & $2,782,669$ & 653 & $2,818,287$ & 8.42 & 101.28 \\
\hline $200-20-5-40$ & 7784 & $2,828,102$ & 659 & $2,865,716$ & 8.46 & 101.33 \\
\hline $200-22-5-50$ & 8647 & $2,791,420$ & 712 & $2,830,779$ & 8.23 & 101.41 \\
\hline $200-25-6-50$ & 8679 & $2,879,922$ & 724 & $2,921,393$ & 8.34 & 101.44 \\
\hline
\end{tabular}

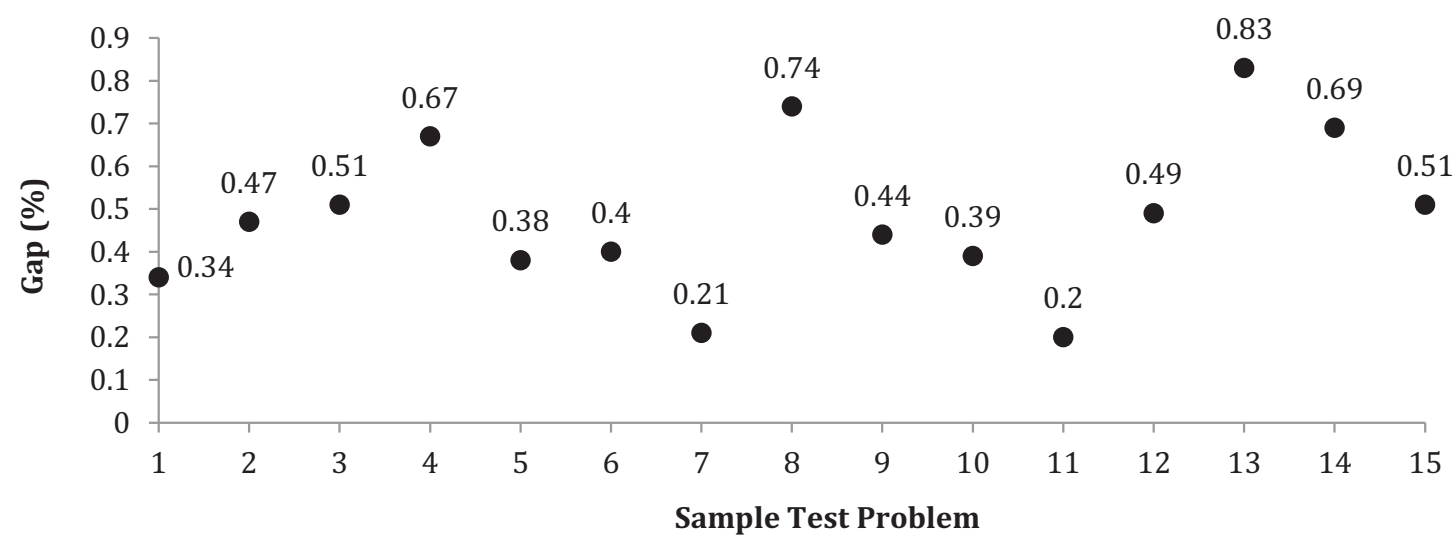

Fig. 8. Gap between LB and optimal solutions. 


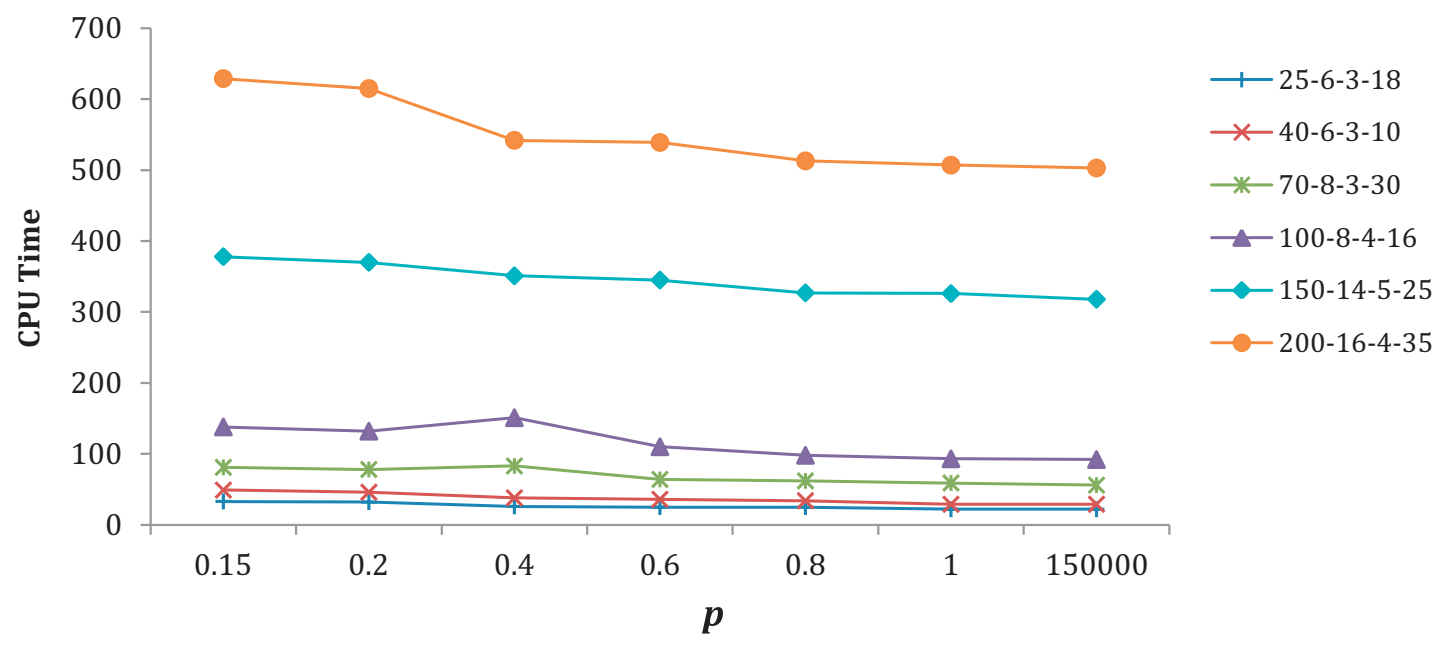

Fig. 9. Algorithm performance vs. different $p$ values.

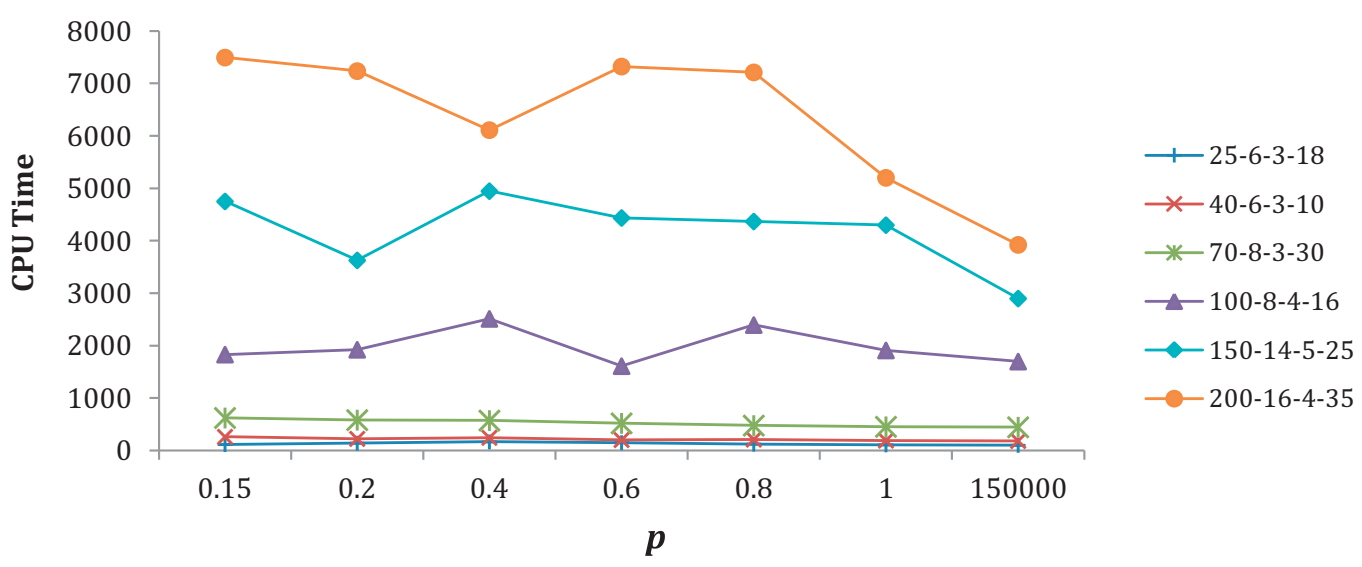

Fig. 10. LB performance vs. different $p$ values.

We also tested the proposed SAICA using instances with smaller disruption probabilities (3\%, $5 \%$, and $10 \%)$, but there was no significant difference in performance. Therefore, the detailed results are omitted due to space limitations.

We also investigated the effect of changing the value of $p$ on the performance of the proposed SAICA. Thus, we performed several numerical experiments with different values of $p$ using various data sets. As shown in Fig. 9, the performance of SAICA was affected little by the value of $p$ for all of the test data sets. However, the CPU time required was higher when the value of $p$ was smaller. In contrast to SAICA, the performance of LB was affected greatly by different values of $p$, as shown in Fig. 10. Therefore, the proposed SAICA method performed efficiently with smaller and larger values of $p$.

\subsection{Minimax cost and minimax regret criteria}

Two other minimax cost and minimax regret robustness measures are often criticized as being too conservative when they are measured against a worst-case scenario that occurs with a small probability [2]. Thus, the relative regrets are increased greatly in other scenarios. In reality, managers are eager to maximize profits and minimize costs, and thus they are not prepared only for the worst situation. The proposed robust approach is more suitable for organizations that plan for the minimum cost or maximum profit over a long-term horizon, while limiting the scenario costs attempts to decrease the short-term effect of disruption. In order to demonstrate that our proposed robust approach is less conservative compared with the minimax cost and minimax regret measures, the results of several experiments are presented in Table 4 . These minimax cost and minimax regret measures were calculated according to models (43) and (44), and solved to optimality using the BARON solver.

Minimax Cost $\quad \min \max _{\omega \in \Psi} \zeta_{\omega}(\boldsymbol{X}, \boldsymbol{Z}, \boldsymbol{U}, \boldsymbol{L}, \boldsymbol{P})$

(12), (13), (16) - (21), (31), (32), (35), (37), (41), (42), 
Table 4

Comparison with the minimax cost and minimax regret criteria.

\begin{tabular}{|c|c|c|c|c|c|c|c|c|c|}
\hline \multirow[t]{2}{*}{$\omega$} & \multirow[t]{2}{*}{$\zeta_{\omega} *$} & \multicolumn{2}{|l|}{$p$-Robust } & \multicolumn{3}{|c|}{ Minimax cost } & \multicolumn{3}{|c|}{ Minimax regret } \\
\hline & & OFV & Regret & OFV & $\%$ GAP & Regret & OFV & $\%$ GAP & Regret \\
\hline 0 & 311,317 & 312,935 & 1.0052 & 335,195 & 7.11 & 1.0767 & 315,768 & 0.91 & 1.0143 \\
\hline 1 & 291,469 & 295,724 & 1.0146 & 338,686 & 14.86 & 1.1620 & 299,018 & 1.11 & 1.0259 \\
\hline 2 & 304,118 & 307,919 & 1.0125 & 329,329 & 6.95 & 1.0829 & 317,286 & 3.04 & 1.0433 \\
\hline 3 & 321,555 & 324,545 & 1.0093 & 337,504 & 3.99 & 1.0496 & 328,307 & 1.16 & 1.0210 \\
\hline 4 & 305,852 & 315,578 & 1.0318 & 314,935 & -0.20 & 1.0297 & 315,241 & -0.11 & 1.0307 \\
\hline 5 & 298,270 & 300,208 & 1.0065 & 332,839 & 10.86 & 1.1159 & 309,216 & 3.00 & 1.0367 \\
\hline 6 & 300,729 & 303,826 & 1.0103 & 321,449 & 5.80 & 1.0689 & 313,389 & 3.15 & 1.0421 \\
\hline 7 & 307,100 & 320,489 & 1.0436 & 319,414 & -0.33 & 1.0401 & 323,130 & 0.82 & 1.0522 \\
\hline 8 & 325,628 & 327,223 & 1.0049 & 354,218 & 14.22 & 1.0878 & 346,370 & 5.85 & 1.0637 \\
\hline 9 & 348,628 & 370,731 & 1.0634 & 360,516 & -2.75 & 1.0341 & 365,292 & -1.46 & 1.0478 \\
\hline 10 & 298,331 & 307,937 & 1.0322 & 324,912 & 5.51 & 1.0891 & 306,326 & -0.52 & 1.0268 \\
\hline 11 & 301,444 & 306,749 & 1.0176 & 333,155 & 8.61 & 1.1052 & 319,018 & 3.99 & 1.0583 \\
\hline 12 & 331,529 & 334,114 & 1.0078 & 349,564 & 4.62 & 1.0544 & 344,723 & 3.17 & 1.0398 \\
\hline 13 & 294,720 & 297,991 & 1.0111 & 317,118 & 6.42 & 1.0760 & 307,127 & 3.06 & 1.0421 \\
\hline 14 & 299,138 & 306,048 & 1.0231 & 311,911 & 1.92 & 1.0427 & 309,099 & 0.99 & 1.0333 \\
\hline
\end{tabular}

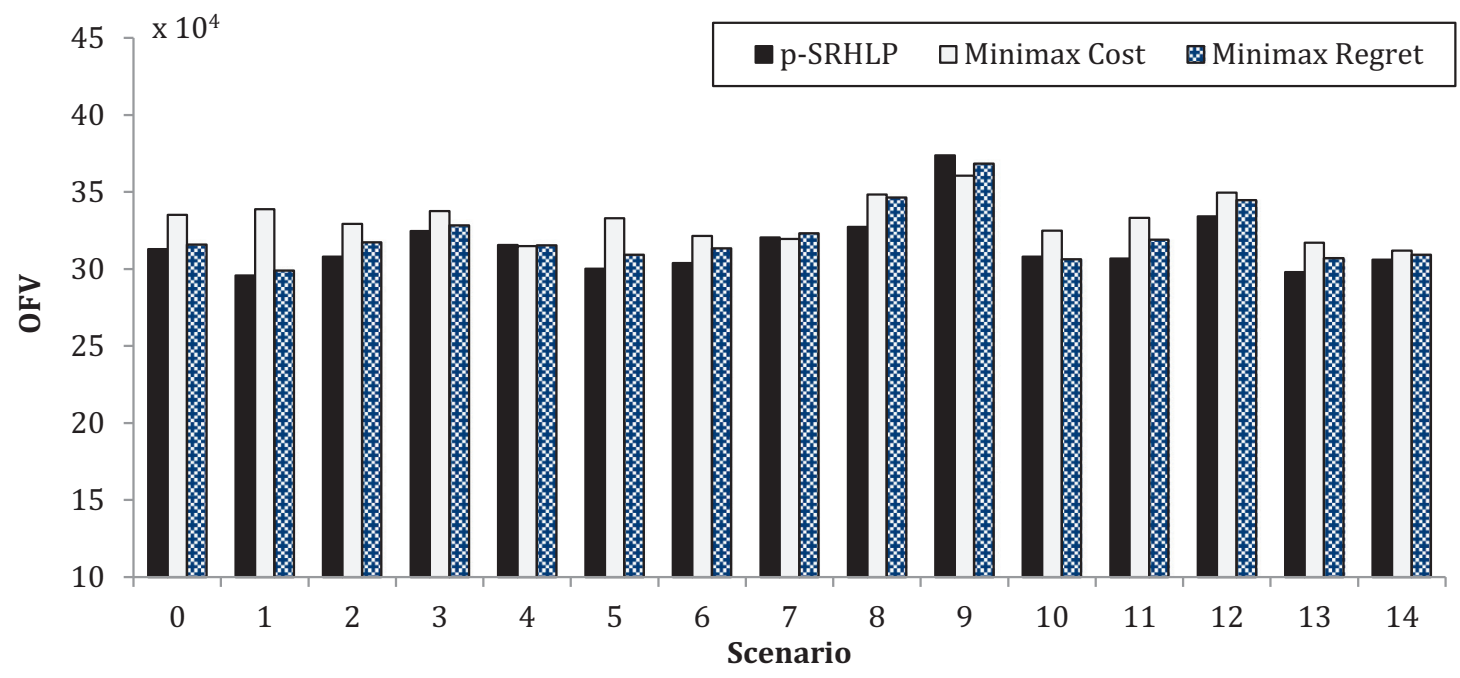

Fig. 11. Comparison between three robustness measures: OFV.

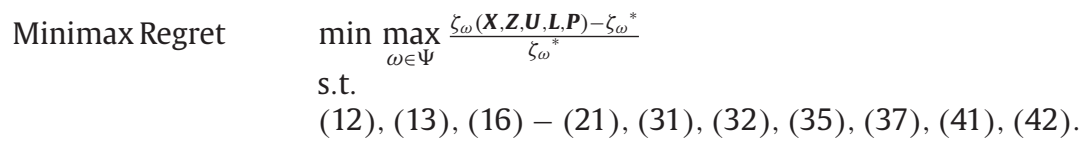

The numerical experiments were conducted using a set of randomly generated data with a size of 15-5-3-14 and $p=0.15$. Table 4 shows the optimal scenario costs $\zeta_{\omega *}$, as well as the scenario cost ("OFV") and relative regret ("Regret") for each model ( $p$-SRHLP, minimax cost, and minimax regret) in each scenario. The percentage differences between the scenario costs for $p$-SRHLP are also compared with the minimax cost and minimax regret models in the "\% GAP" column. The costs and regrets of each model for each scenario are depicted in Figs. 11 and 12, respectively. Table 4 and Figs. 11 and 12 show that the minimax cost and regret models mostly considered the worst-case scenario (i.e., scenario 9) in this data set. In contrast to the minimax models, the proposed p-SRHLP model had a higher cost in scenario 9, but lower costs in all but three of the other scenarios.

In addition, the relative regrets were very large in the minimax cost model ( $\geq 14 \%$ ) compared with the $p$-SRHLP and minimax regret models. Moreover, the $p$-SRHLP model obtained smaller relative regrets compared with the minimax regret model in most of the scenarios, while it tried to minimize the cost of the nominal scenario and it was affected less by the disruption scenarios. Thus, the proposed $p$-SRHLP was less conservative than the other two models. Furthermore, another advantage of the $p$-SRHLP model is its flexibility in terms of adjusting the value of $p$ to obtain different solutions, whereas the other two models provide only one solution. 


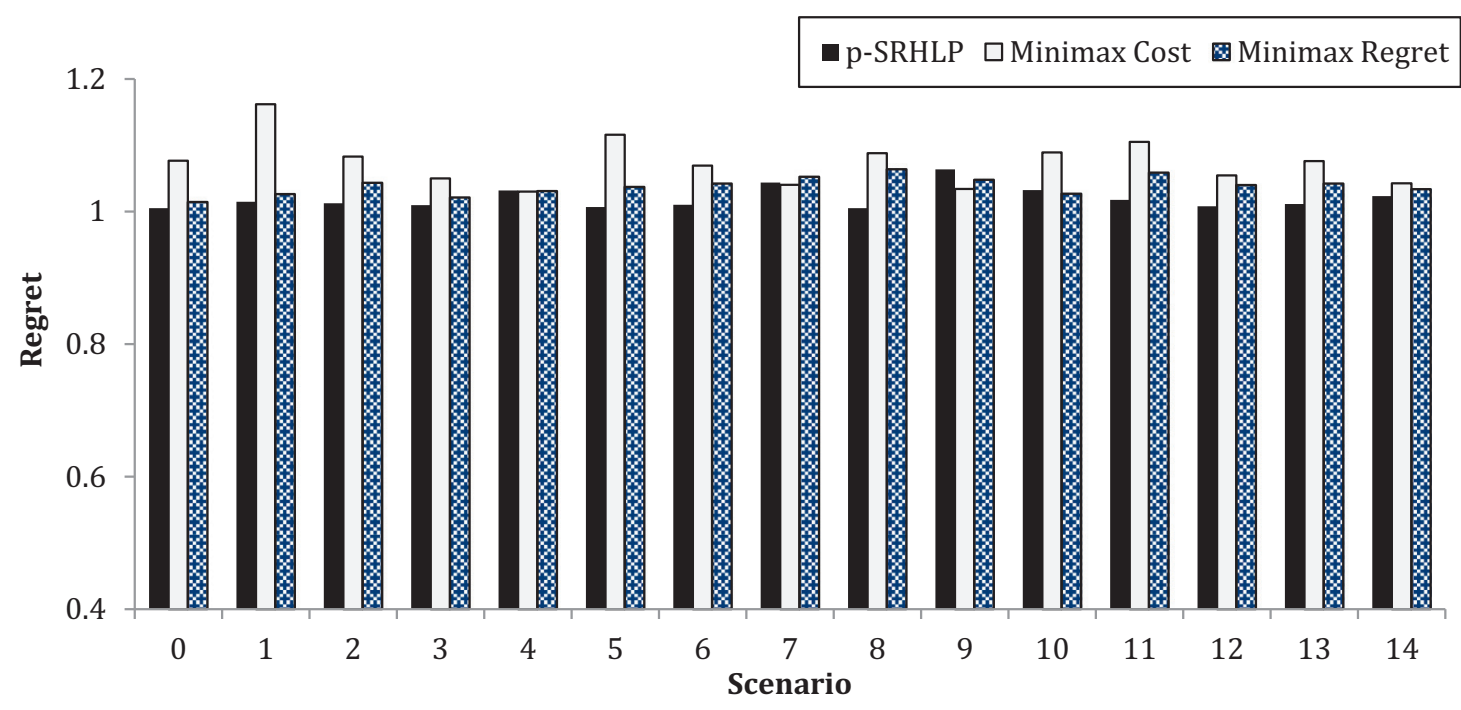

Fig. 12. Comparison between three robustness measures: Regret.

Table 5

OFV vs. the maximum regret.

\begin{tabular}{llllll}
\hline Test problem & $p$ & OFV & OFV inc (\%) & Max regret & Max regret dec (\%) \\
\hline 25-6-3-18 & $\infty$ & 348,110 & 0 & 0.8535 & 0 \\
& 0.8535 & 352,537 & 1.27 & 0.7245 & 17.80 \\
& 0.7245 & 353,371 & 0.23 & 0.6475 & 11.89 \\
& 0.6475 & 354,112 & 0.21 & 0.6015 & 7.64 \\
& 0.6015 & 354,714 & 0.17 & 0.5725 & 5.06 \\
& 0.5725 & 355,224 & 0.14 & 0.5485 & 4.37 \\
& 0.5485 & 357,890 & 0.75 & 0.5265 & 4.18 \\
& 0.5265 & 359,474 & 0.44 & 0.5045 & 4.36 \\
& 0.5045 & 360,331 & 0.23 & 0.4985 & 1.20 \\
& 0.4985 & 361,668 & 0.37 & 0.4945 & 0.81 \\
& 0.4945 & 362,777 & 0.31 & 0.4915 & 0.61 \\
& 0.4915 & 364,482 & 0.47 & 0.4905 & 0.20 \\
& $\infty$ & 629,311 & 0 & 0.7135 & 0 \\
& 0.7135 & 635,629 & 1.0 & 0.6355 & 12.27 \\
& 0.6355 & 636,003 & 0.06 & 0.5585 & 13.78 \\
& 0.5585 & 636,667 & 0.11 & 0.4845 & 15.27 \\
& 0.4845 & 637,101 & 0.07 & 0.4315 & 12.28 \\
& 0.4315 & 638,193 & 0.17 & 0.4045 & 6.67 \\
& 0.4045 & 639,722 & 0.24 & 0.3725 & 8.59 \\
0.3725 & 640,110 & 0.06 & 0.3515 & 5.97 \\
& 0.3515 & 641,403 & 0.20 & 0.3485 & 0.86 \\
& 0.3485 & 643,220 & 0.28 & 0.3445 & 1.16 \\
& 0.3445 & 644,339 & 0.17 & 0.3425 & 0.58 \\
& 0.3425 & 646,208 & 0.29 & 0.3405 & 0.58 \\
\hline
\end{tabular}

\subsection{Price of robustness}

One of the most important issues in the proposed p-SRHLP is the "price of robustness," i.e., the extra cost required to design a more robust and reliable hub network with much lower scenario costs when disruptions occur. According to Snyder and Daskin [13], more robust solutions can be found with a small increase in the cost. In this section, we show that our proposed modeling approach also obtains similar trends. Thus, we investigated the trade-off between the maximal relative regret and the nominal cost as follows. First, we considered $p=1$ and solved the problem, and we calculated the maximum relative regret over all the scenarios. Next, we set $p$ to the maximum relative regret minus 0.0005 and re-solved the problem. This procedure was repeated by reducing $p$ at each iteration and we re-solved the problem until no feasible solution could be found. With further iterations, the objective value increased while the maximum regret decreased when the $p$-robustness constraints were tightened.

We applied this procedure to two test problems, "25-6-3-18" and "70-8-3-30," and the results are reported in Table 5. The column " $p$ " shows different values of $p$. The third column ("OFV") shows the objective function value obtained by the 


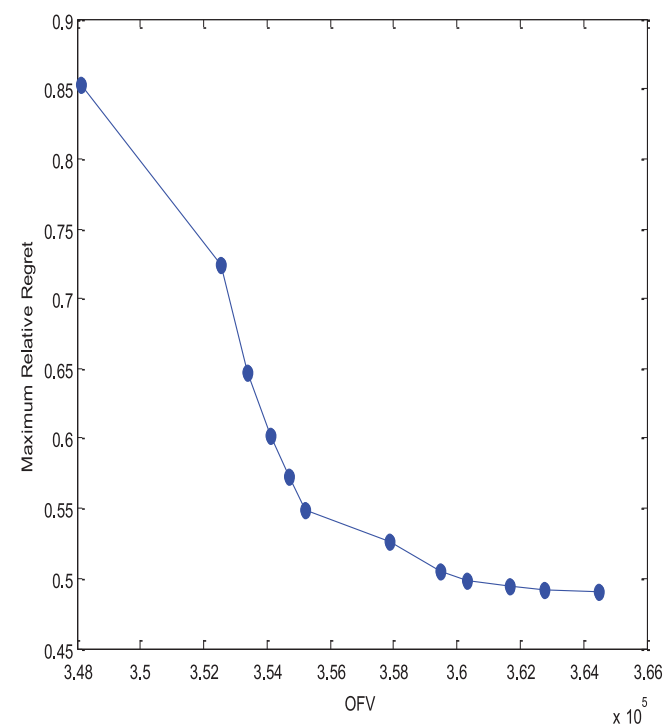

a) Test problem: "25-6-3-18"

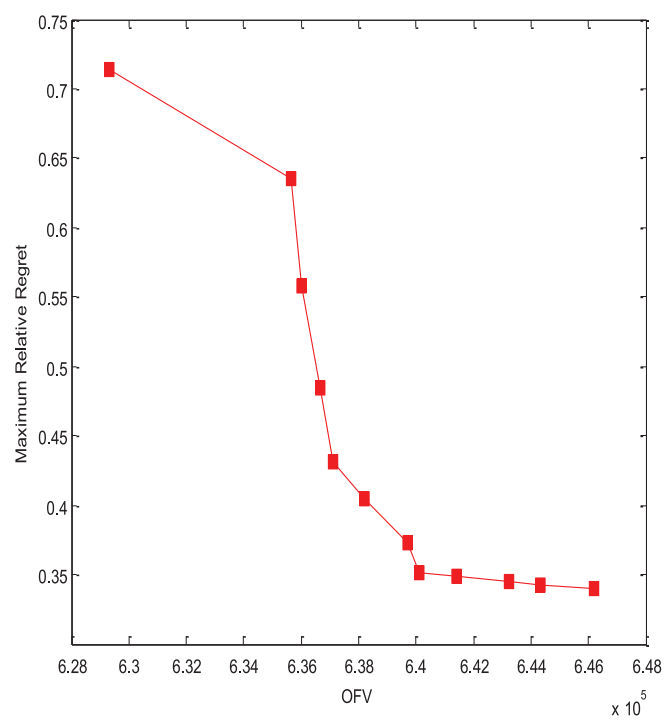

b) Test problem: “70-8-3-30"

Fig. 13. Comparison between three robustness measures: Regret.

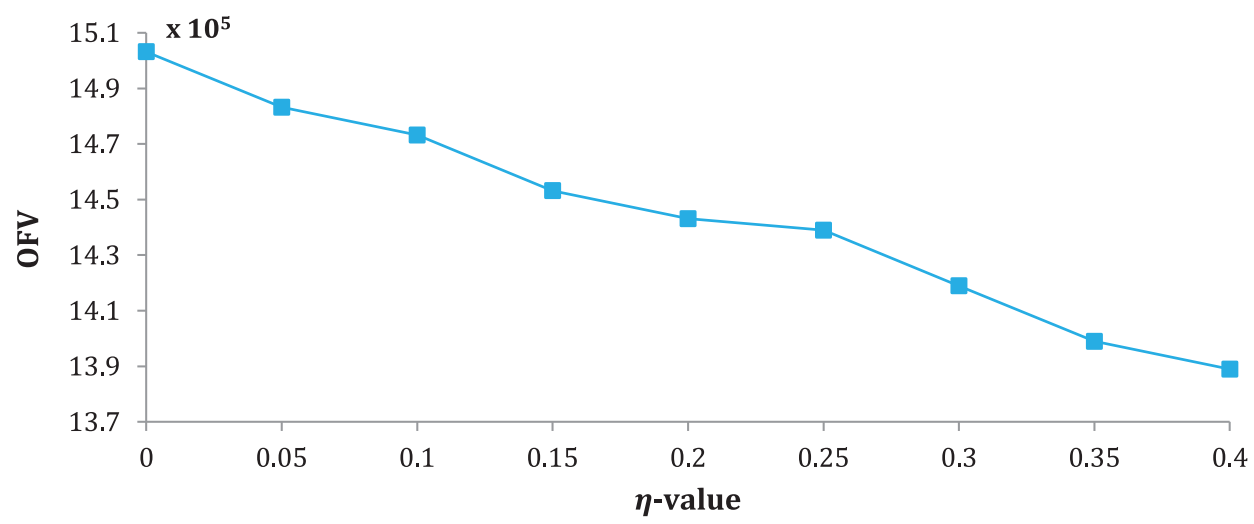

Fig. 14. OFV and capacity exceedance probability.

proposed SAICA. The fourth column shows the percentage increase in the OFV with respect to the decrease in the value of $p$. The fifth column presents the maximum relative regret. The last column shows the percentage decrease in the maximum relative regret with respect to the increase in the value of $p$. It should be noted that the only first 12 solutions are reported in Table 5, but they provide the minimum OFV. The trade-off curves between the objective value and maximum relative regret for both test problems are illustrated in Fig. 13.

According to Table 5 and Fig. 13, it can be seen that a high decrease in the maximum relative regret could be obtained with only a small increase in the nominal cost. For example, for test problem "25-6-3-18," a considerable decrease in the maximum relative regret was obtained of $40.89 \%$ from 0.8535 to 0.5045 , and with a slight increase in the cost of $2.21 \%$ from 352,537 to 360,331 . Thus, we could obtain a robust hub network that was more reliable with a slight increase in the total cost in the nominal scenario.

\subsection{Sensitivity analyses}

We conducted a sensitivity analysis to further validate the efficiency and effectiveness of the model, as well as determining the degree of influence for the parameters employed in the model, especially the capacity exceedance probability $(\theta)$ and the capacity disruption factor $(\eta)$. It should be noted that the sensitivity analysis was performed for test problem "100-16-4-45" with $p=0.25$. Fig. 14 illustrates the results of the sensitivity analysis for the capacity exceedance probability. Hubs with a lower capacity had a lower cost, so hubs with a lower capacity could be located by increasing the exceedance probability of the capacity; therefore, the total cost of the hub network decreased. In addition, Fig. 15 shows the sensitivity 


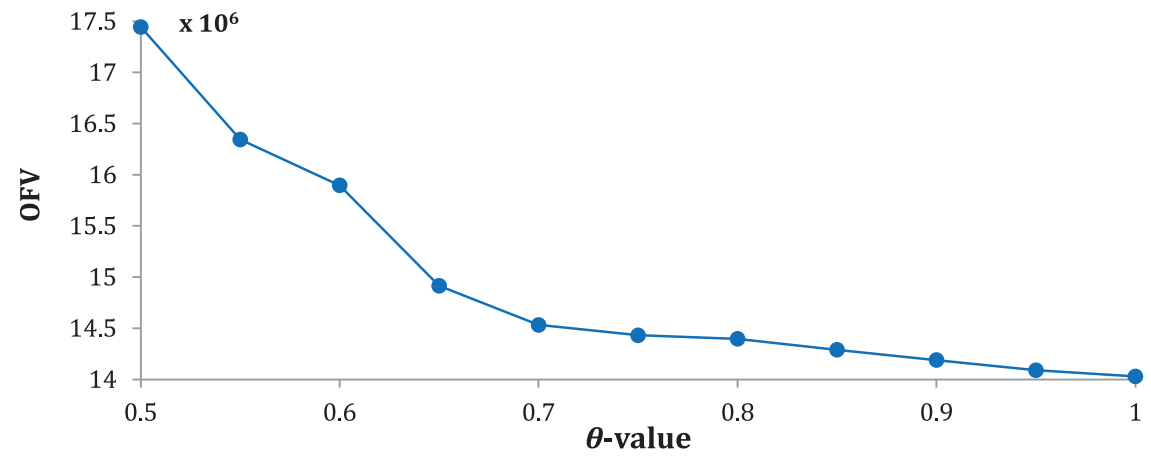

Fig. 15. OFV and capacity disruption factor.

of the objective function value versus the capacity disruption factor, which indicates that by increasing the disruption factor, the total cost was decreased when hubs with lower capacity and lower costs could be located.

\section{Conclusion}

In this study, we considered a strategic supply chain management problem to design reliable logistics networks based on a HLP, which perform as well as possible under normal conditions, while also performing relatively well when disruptions occur. We formulated the $p$-SRHLP in order to minimize the nominal cost subject to the constraint that the solution must have a relative regret no greater than $p$ in each scenario. We also developed a new self-adaptive meta-heuristic algorithm called SAICA based on genetic algorithms and ICA to solve the presented model. Our computational experiments showed that we could obtain quality solutions, which were very close to optimal, in a fraction of the time required by the BARON solver. We performed several numerical experiments, which showed that the proposed approach could obtain less conservative solutions compared with traditional robustness criteria (e.g., minimax cost and minimax regret). Furthermore, we obtained some important managerial insights because the robustness could be increased considerably without any significant increase in the total cost. Our results also indicated that the reliability could be improved by increasing the flexibility of the hub network. In addition, a trade-off curve generated by altering the value of $p$ could help decision makers to choose the desired robustness level based on budget constraints.

\section{Acknowledgments}

This work has been supported financially by the Center for International Scientific Studies \& Collaboration (CISSC) and the French Embassy in Tehran as well as the Partenariats Hubert Curien (PHC) program in France. Additionally, the authors would like thank the Editor-in-Chief and anonymous reviewers for their valuable comments and acceptance letter.

\section{References}

[1] T. Cui, Y. Ouyang, Z.J.M. Shen, Reliable facility location design under the risk of disruptions, Oper. Res. 58 (4) (2010) $998-1011$.

[2] P. Peng, L.V. Snyder, Z. Liu, A. Lim, Design of reliable logistics networks with facility disruptions, Transp. Res. Part B Methodol. 45 (2011) $1190-1211$.

[3] B.C. Tansel, R.L. Francis, T.J. Lowe, Location on networks: a survey. Part I: the P-center and P-median problems, Manage. Sci. 29 (1983) $482-497$.

[4] G.L. Nemhauser, L.A. Wolsey, Integer and Combinatorial Optimization, Wiley-Interscience, 1988.

[5] L.V. Snyder, Facility location under uncertainty: a review, IIE Trans. 38 (2006) 537-554.

[6] Z. Drezner, G. Wesolowsky, Network design: selection and design of links and facility location, Transp. Res. Part A 37 (3) (2003) $241-256$.

[7] L.V. Snyder, M.S. Daskin, Reliability models for facility location: the expected failure cost case, Transp. Sci. 39 (2005) $400-416$.

[8] O. Berman, D. Krass, M.B.C. Menezes, Facility reliability issues in network p-median problems: strategic centralization and co-location effects, Oper. Res. 55 (2) (2007) 332-350.

[9] X. Li, Y. Ouyang, A continuum approximation approach to reliable facility location design under correlated probabilistic disruptions, Transp. Res. Part B 44 (4) (2010) 535-548.

[10] M. Lim, M.S. Daskin, A. Bassamboo, S. Chopra, Facility location decisions in supply chain networks with random disruption and imperfect information, in: Working Paper, Department of Industrial Engineering and Management Sciences, Northwestern University, Evanston, IL, 2009.

[11] M. Lim, M.S. Daskin, A. Bassamboo, S. Chopra, A facility reliability problem: formulation, properties and algorithm, Naval Res. Logist. 57 (2010) 58-70.

[12] Z.-J.M. Shen, R.L. Zhan, J. Zhang, The reliable facility location problem: formulations, heuristics, and approximation algorithms, in: Working Paper, Department of Industrial Engineering and Operations Research, University of California at Berkeley, 2007.

[13] L. Snyder, M. Daskin, Stochastic p-robust location problems, IIE Trans. 38 (11) (2006) 971-985.

[14] R.L. Zhan, M.S. Daskin, Z.-J.M. Shen, Facility reliability with site-specific failure probabilities, in: Working Paper, Department of Industrial Engineering and Operations Research, University of California-Berkeley, 2008.

[15] Q. Li, B. Zeng, A. Savachkin, Reliable facility location design under disruptions, Comput. Oper. Res. 40 (2013) 901-909.

[16] F. Parvaresh, S.A. Hashemi Golpayegany, S.M. Moattar Husseini, B. Karimi, Solving the p-hub median problem under intentional disruptions using simulated annealing, Network Spatial Econ. 13 (4) (2013) 445-470.

[17] F. Parvaresh, S.M. Moattar Husseini, S.A. Hashemi Golpayegany, B. Karimi, Hub network design problem in the presence of disruptions, J. Intell. Manuf. 25 (4) (2014) 755-774.

[18] Mohammadi, M., Tavakkoli-Moghaddam, R., 2015. Design of a fuzzy bi-objective reliable p-hub center problem. J. Intell. Fuzzy Syst. (in press), doi:10. 3233/IFS-151846. 
[19] H. Calik, S.A. Alumur, B.Y. Kara, O.E. Karasan, A tabu-search based heuristic for the hub covering problem over incomplete hub networks, Comput. Oper. Res. 36 (2009) 3088-3096.

[20] I. Contreras, E. Fernandez, A. Marín, The tree of hubs location problem, Eur. J. Oper. Res. 202 (2010) 390-400.

[21] H. Lo, X.W. Luo, W.Y. Siu, Degradable transport network: travel time budget of travelers with heterogeneous risk aversion, Transp. Res. Part B 40 (2006) 792-806.

[22] H. Lo, Y.K. Tung, Network with degradable links: capacity analysis and design, Transp. Res. Part B 37 (4) (2003) $345-363$.

[23] A. Chen, H. Yang, H. Lo, W.H. Tang, Capacity reliability of a road network: an assessment methodology and numerical results, Transp. Res. Part B 36 (3) (2002) 225-252

[24] X.W. Luo, Degradable Transport Networks MPhil. thesis), Department of Civil Engineering, Hong Kong University of Science and Technology, Hong Kong, 2004.

[25] M. Mohammadi, F. Jolai, H. Rostami, An M/M/c queue model for hub covering location problem, Math. Comput. Modell. 54 (2011) $2623-2638$.

[26] M. Mohammadi, F. Jolai, R. Tavakkoli-Moghaddam, Solving a new stochastic multi-mode p-hub covering location problem considering risk by a novel multi-objective algorithm, Appl. Math. Model. 37 (2013) 10053-10073.

[27] M. Mohammadi, S.A. Torabi, R. Tavakkoli-Moghaddam, Sustainable hub location under mixed uncertainty, Transp. Res. Part E 62 (2014) 89-115.

[28] M. Mohammadi, S. Dehbari, B. Vahdani, Design of a bi-objective reliable healthcare network with finite capacity queue under service covering uncertainty, Transp. Res. Part E 72 (2014) 15-41.

[29] F. Niakan, B. Vahdani, M. Mohammadi, A multi-objective optimization model for hub network design under uncertainty: an inexact rough-interval fuzzy approach, Eng. Optim. (2015), doi:10.1080/0305215X.2014.992891.

[30] B. Zahiri, R. Tavakkoli-Moghaddam, M. Mohammadi, P. Jula, Multi-objective design of an organ transplant network under uncertainty, Transp. Res. Part E 72 (2014) 101-124.

[31] I. Contreras, J.F. Cordeau, G. Laporte, Stochastic uncapacitated hub location, Eur. J. Oper. Res. 212 (2011) $518-528$.

[32] R. Ishfaq, C.R. Sox, Design of intermodal logistics networks with hub delays, Eur. J. Oper. Res. 220 (2012) 629-641.

[33] B. Zahiri, R. Tavakkoli-Moghaddam, M.S. Pishvaee, A robust possibilistic programming approach to multi-period location-allocation of organ transplant centers under uncertainty, Comput. Ind. Eng. 74 (2014) 139-148.

[34] B. Zahiri, S.A. Torabi, M. Mousazadeh, S.A. Mansouri, Blood collection management: methodology and application, Appl. Math. Modell. 39 (23) (2015) $7680-7696$.

[35] P. Kouvelis, A. Kurawarwala, G. Gutiérrez, Algorithms for robust single and multiple period layout planning for manufacturing systems, Eur. J. Oper. Res. 63 (2) (1992) 287-303.

[36] A. Kleywegt, A. Shapiro, T. Homem-De-Mello, The sample average approximation method for stochastic discrete optimization, SIAM J. Optim. 12 (2) (2001) 479-502.

[37] H. Prüfer, Neuer Beweis eines Satzes ueber Permutationen, Arch. Math. Phys. 27 (1918) 742-744

[38] M. Mohammadi, R. Tavakkoli-Moghaddam, A. Ghodratnama, H. Rostami, Genetic and improved shuffled frog leaping algorithms for a 2-stage model of a hub covering location network, Int. J. Ind. Eng. Prod. Res. 22 (3) (2011) 179-187.

[39] M. Mohammadi, A. Siadat, J.Y. Dantan, R. Tavakkoli-Moghaddam, Mathematical modelling of a robust inspection process plan: Taguchi and Monte Carlo methods, Int. J. Prod. Res. 53 (7) (2015) 2202-2224.

[40] R. Ghodsi, M. Mohammadi, H. Rostami, Hub covering location problem under capacity constraints, in: 2010 Fourth Asia International Conference on Mathematical/Analytical Modelling and Computer Simulation (AMS), IEEE, 2010, May, pp. 204-208.

[41] R. Azizmohammadi, M. Amiri, R. Tavakkoli-Moghaddam, M. Mohammadi, Solving a redundancy allocation problem by a hybrid multi-objective imperialist competitive algorithm, Int. J. Eng. Trans. C Aspects 26 (9) (2013) 1031.

[42] E. Atashpaz-Gargari, C. Lucas, Imperialist competitive algorithm: an algorithm for optimization inspired by imperialist competitive, in: Proceedings of the IEEE Congress on Evolutionary Computation, CEC, Singapore, 2007.

[43] S.N. Sivanandam, S.N. Deepa, Introduction to Genetic Algorithms, Springer, 2008.

[44] R. Aboolian, T. Cui, Z.J. Max Shen, An efficient approach for solving reliable facility location models, INFORMS J. Comput. 25 (4) (2012) 720-729. 